\title{
The Neural Correlates of Consciousness
}

\author{
An Update
}

\author{
GiUlio TonONI $^{a}$ AND GHRISTOF KOCH ${ }^{b}$ \\ ${ }^{a}$ Department of Psychiatry, University of Wisconsin, Madison, Wisconsin, USA \\ ${ }^{b}$ Division of Biology and the Division of Engineering and Applied Science, California \\ Institute of Technology, Pasadena, California, USA
}

\begin{abstract}
This review examines recent advances in the study of brain correlates of consciousness. First, we briefly discuss some useful distinctions between consciousness and other brain functions. We then examine what has been learned by studying global changes in the level of consciousness, such as sleep, anesthesia, and seizures. Next we consider some of the most common paradigms used to study the neural correlates for specific conscious percepts and examine what recent findings say about the role of different brain regions in giving rise to consciousness for that percept. Then we discuss dynamic aspects of neural activity, such as sustained versus phasic activity, feedforward versus reentrant activity, and the role of neural synchronization. Finally, we briefly consider how a theoretical analysis of the fundamental properties of consciousness can usefully complement neurobiological studies.
\end{abstract}

Key words: consciousness; brain; information

\section{Introduction}

There are several strategies by which scientists can approach the relationship between the brain and consciousness. One is to wait and hope that, once we know all there is to know about neuroanatomy and neurophysiology (or maybe when we can model in detail the working of the entire brain; Markram 2006), the answer will somehow pop out. A more practical approach is to use the tools of neuroscience that are available now to shed light on the neural structures and activity patterns that underlie consciousness. For example, one can examine how brain activity changes when, everything else being as equal as possible, a stimulus is experienced or not. In this way, one can attempt to identify, more and more precisely, the neural correlates of consciousness (NCG), defined as the minimal neuronal mechanisms jointly sufficient for any one specific conscious percept (Koch 2003). A complementary strategy is to consider conditions in which consciousness is globally diminished, such as deep sleep or anesthesia, and ask what has changed in the brain. Finally, another approach is to develop a theoretical framework that clarifies what

Address for correspondence: Christof Koch, Division of Biology and the Division of Engineering and Applied Science, 216-76, California Institute of Technology, Pasadena, CA 91125. Fax: +1-626-796-8876.

koch@klab.caltech.edu consciousness is, how it can be generated by a physical system, and how it can be measured and then to test the predictions of the theory against data from neuroscience (Tononi 2004b).

This review focuses on recent advances in the study of the NCC. First, we briefly discuss some useful distinctions between consciousness and other brain functions. We then examine what has been learned about the NCG by studying global changes in the level of consciousness, such as sleep, anesthesia, and generalized seizures. Next, we consider some of the most common paradigms used to study the NCG for specific conscious percepts and examine what recent findings say about the role of different brain regions in giving rise to consciousness for that percept. Finally, we discuss the relation to the NCG of dynamic aspects of neural activity, such as sustained versus phasic activity, feedforward versus reentrant activity, and the role of neural synchronization. We end with a few remarks on the importance of a combined theoretical and neurobiological approach.

\section{Consciousness and Other Brain Functions}

Many suggestions have been ventured in the hope of alleviating the puzzle of subjective experience. Perhaps consciousness emerges somehow when an organism is 
immersed in some complex sensorimotor loop that includes the environment. Another common idea is that consciousness may somehow arise when one part of the brain, acting as the "subject" (typically the front), looks upon another part as its "object" (typically the back), and evaluates or reflects upon its activity. It is often thought that in the end consciousness may reduce to attention and its brain mechanisms, since we are usually conscious of what we attend. Much could be said about each of these suggestions. Here, we briefly consider some recent results (and some very old evidence) indicating that consciousness - in the sense of having an experience - does not require sensorimotor loops involving the body and the world, does not require self-reflection (or language), and does not reduce to attention.

\section{Consciousness and Sensory Input/Motor Output}

We are usually conscious of what goes on around us, and occasionally of what goes on within our body. So it is only natural to think that consciousness may be tightly linked to the ongoing interaction we maintain with the world and the body. However, there are many examples to the contrary. We are conscious of our thoughts, which do not seem to correspond to anything out there; we can also imagine things that are not out there. When we do so, sensory areas can be activated from the inside (Kosslyn et al. 2001), though there are some differences (Amedi et al. 2005). Also, stimulus-independent consciousness is associated with its own patterns of activation within cortex and thalamus (Mason et al. 2007). During dreams, we are virtually disconnected from the environment (Hobson et al. 2000) - hardly anything of what happens around us enters consciousness, and our muscles are paralyzed (except for eye muscles and diaphragm). Nevertheless, we are vividly conscious: all that seems to matter is that the thalamocortical system continues to function more or less as in wakefulness, as shown by unit recording, EEG, and neuroimaging studies performed during rapid eye movement (REM) sleep, when dreams are most intense (Maquet et al. 1996). Interestingly, certain regions of the thalamocortical systems, such as dorsolateral prefrontal cortex, are deactivated in REM sleep, which likely accounts for some peculiarities of dreaming experiences, such as the reduction of voluntary control.

Neurological evidence also indicates that neither sensory inputs nor motor outputs are needed to generate consciousness. For instance, retinally blind people can both imagine and dream visually if they become blind after 6-7 years of age or so (Hollins 1985; Buchel et al. 1998). Patients with the locked-in syndrome can be almost completely paralyzed, and yet they are just as conscious as healthy subjects (Laureys et al. 2005) and can compose eloquent accounts of their condition (Bauby 1997). A transient form of paralysis is one of the characteristic features of narcolepsy. Severe cataleptic attacks can last for minutes and leave the patient collapsed on the floor, utterly unable to move or to signal, but fully aware of her surroundings (Guilleminault 1976; Siegel 2000). Or consider the Californian drug addicts known as the frozen addicts who acquired some of the symptoms of severe, late-stage Parkinson's disease, fully conscious, yet unable to move or speak (Langston \& Palfreman 1995). All six had previously taken synthetic heroin tainted with MPTP, which selectively and permanently destroyed dopamine-producing neurons in their basal ganglia. Thus, consciousness here and now seems to depend on what certain parts of the brain are doing, without requiring any obligatory interaction with the environment or the body.

\section{Consciousness and Self-Reflection}

Consciousness is usually evaluated by verbal reports, and questions about consciousness ("Did you see anything on the screen?") are answered by "looking inside" retrospectively and reporting what one has just experienced. So it is perhaps natural to suggest that consciousness may arise through the ability to reflect on our own perceptions: our brain would form a scene of what it sees, but we would become conscious of itexperience it subjectively - only when we, as a subject of experience, watch that scene from the inside. This suggestion is often framed in a neurobiological context by assuming that patterns of activity corresponding to "unconscious" or "subconscious" percepts form in posterior regions of the cerebral cortex involved in the categorization/association of sensory stimuli. These percepts then become conscious when mainly anterior prefrontal and cingulate regions involved in selfrepresentations interact with posterior cortex, perhaps by reading in signals through forward connections and selectively amplifying them through back connections (more on this later).

There is of course no doubt that the brain categorizes its own patterns of activity in the sense that neurons respond mainly to the activity of other neurons, so the brain is constantly "looking at itself." However, this is not necessarily in terms of a "subject" (the front) looking at an "object" represented in sensory cortices (the back). Leaving aside the mystery of why reflecting on something should make it conscious, this scenario is made less plausible by a common observation: when we become absorbed in some intense perceptual task, 
for example watching an engrossing movie, playing a fast-paced video game, or rushing through the woods at high speed, we are vividly conscious - we are immersed in the rapid flow of experience - without any need for reflection or introspection. Often, we become so immersed in such flow that we may lose the sense of self, the inner voice. Perhaps the habit of thinking about consciousness has distracted the scholars who write upon such matters to devalue the unreflective nature of much of experience.

A recent neuroimaging study by Malach and collaborators (Hasson et al. 2004) throws some interesting light on these old observations. Subjects were scanned with fMRI in three conditions. In the slow categorization task, subjects were asked to categorize pictures into animal/no-animal categories. During the introspective task, subjects viewed the images and then introspected about their emotional response (strong/neutral). Finally, the fast categorization task was identical to the "slow" condition but at triple the stimulation rate. Thus, "slow" and "introspection" conditions were identical in terms of sensory stimuli and motor output but differed in the cognitive task. On the other hand, "slow" and "rapid" conditions were similar in the cognitive task but differed in the sensorimotor processing and attentional loads. Behavioral measurements confirmed that self-awareness was high during the introspection task, and virtually abolished during rapid categorization. The neuroimaging results were clear: during introspection there was an activation of prefrontal regions, whereas sensory cortex was strongly activated during rapid categorization. Crucially, during introspection self-related cortex was deactivated below the rest condition. This deactivation of prefrontal regions was thus the neural correlate of "losing oneself" in the task. To the extent that these prefrontal regions were indeed involved in selfrepresentation, these findings suggest that their activation is not necessary for the emergence of perceptual consciousness, but only to reflect upon it and report it to others. Indeed, it appears that self-related activity is actually shut off during highly demanding sensory tasks.

Lesion studies also seem to support the notion that consciousness does not require prefrontal cortex and, by inference, the functions it performs. Though patients with widespread, bilateral damage to prefrontal cortex are rare, two recent clinical studies provide some intriguing evidence. A man who, at the age of 21, had fallen on an iron spike that completely penetrated through both of his frontal lobes, nevertheless went on to live a stable life - marrying and raising two children - in an appropriate professional and social setting. Although displaying many of the typical frontal lobe behavioral disturbances, he never complained of loss of sensory perception, nor did he show visual or other deficits (Mataro et al. 2001). Another case is that of a 27-year-old woman with massive bilateral prefrontal damage of unclear etiology (Markowitsch \& Kessler 2000). While manifesting grossly deficient scores in frontal lobe sensitive tests, she showed no abnormal perceptual abilities (that is not to say that such patients do not suffer from subtle visual deficits; Barcelo et al. 2000).

\section{Consciousness and Attention}

Consciousness and attention have resisted clear and compelling definitions. Few would dispute that the relationship between the two is an intimate one. When subjects pay attention to an object, they become conscious of its various attributes; when the focus of attention shifts away, the object fades from consciousness. Indeed, more than a century of research efforts have quantified the ample benefits accrued to attended and consciously perceived events (Pashler 1998; Braun et al. 2001). This has prompted many to posit that the two processes are inextricably interwoven, if not identical (Posner 1994; Merikle \& Joordens 1997; Chun \& Wolfe 2000; O'Regan \& Noe 2000). Others, however, going back to the 19th century, have argued that attention and consciousness are distinct phenomena, with distinct functions and neuronal mechanisms (Iwasaki 1993; Hardcastle 1997; Lamme 2003; Baars 2005; Block 2005; Dehaene et al. 2006; Koch \& Tsuchiya 2007). Recent psychophysical and neurophysiological evidence argues in favor of a dissociation between selective attention and consciousness, suggesting that events or objects can be attended to without being consciously perceived. Conversely, an event or object can be consciously perceived in the near absence of top-down attentional processing.

\section{Attention without Consciousness}

Consider that subjects can attend to a location for many seconds and yet fail to see one or more attributes of an object at that location. In lateral masking (visual crowding), the orientation of a peripherally presented grating is hidden from conscious sight but remains sufficiently potent to induce an orientation-dependent aftereffect (He et al. 1996) Montaser-Kouhsari and Rajimehr (2004) showed that an aftereffect induced by an invisible illusory contour required focal attention, even though the object at the center of attention was invisible. Naccache and colleagues (2002) elicited priming for invisible words (suppressed by a combination of forward and backward masking) but only if 
the subject was attending to the invisible prime-target pair; without attention, the same word failed to elicit priming. That is, the subject needs to attend to the masked stimulus for priming to occur, but the actual stimulus is not seen (with an associated $\mathrm{d}^{\prime}=0$ ). In another experiment, male/female nudes attracted attention and induced involuntary eye movements when they were rendered completely invisible by continuous flash suppression (Jiang et al. 2006). When subjects had to discriminate the location of the masked nude from the location of a masked shuffled nude, they were at chance; without the intraocular masking, the images are clearly visible. fMRI evidence confirms attentional modulation of invisible images in primary visual cortex (Bahrami et al. 2007). In conclusion, attentional selection does not necessarily engender phenomenal sensations, although it may often do so.

\section{Consciousness in the Absence of Attention}

When focusing intensely on one event, the world is not reduced to a tunnel, with everything outside the focus of attention gone: we are always aware of some aspects of the world surrounding us, such as its gist. Indeed, gist is immune from inattentional blindness (Mack \& Rock 1998) — when a photograph covering the entire background is briefly and unexpectedly flashed onto the screen, subjects can accurately report a summary of its content. In the $30 \mathrm{~ms}$ necessary to apprehend the gist of a scene, top-down attention cannot play much of a role (because gist is a property associated with the entire image, any process that locally enhances features is going to be of limited use).

Or take the perception of a single object (say a bar) in an otherwise empty display, a nonecological but common arrangement in many animal and human experiments. Here, what function would top-down, selective attention need to perform without any competing item in or around fixation? Indeed, the most popular neuronal model of attention, biased competition (Desimone \& Duncan 1995), predicts that in the absence of competition, no or little attentional enhancement occurs, yet we are perfectly aware of the object and its background.

In a dual-task paradigms, the subject's attention is drawn to a demanding central task, while at the same time a secondary stimulus is flashed somewhere in the periphery. Using the identical retinal layout, the subject either performs the central task, the peripheral task, or both simultaneously (Sperling \& Dosher 1986; Braun \& Sagi 1990; Braun \& Julesz 1998). With focal attention busy at the center, the subject can still distinguish a natural scene containing an animal (or a vehicle) from one that does not include an animal (or a vehicle), while being unable to distinguish a redgreen bisected disk from a green-red one $(\mathrm{Li}$ et al. 2002). Likewise, subjects can tell male from female faces or even distinguish a famous from a nonfamous face (Reddy et al. 2004; Reddy \& Koch 2006) but are frustrated by tasks that are computationally much simpler (e.g., discriminating a rotated letter "L" from a rotated "T"). This is quite remarkable. Thus, while we cannot be sure that observers do not deploy some limited amount of top-down attention in these dual-task experiments that require training and concentration (i.e., high arousal), it remains true that subjects can perform certain discriminations but not others in the near absence of top-down attention. And they are not guessing. They can be quite confident of their choices and "see," albeit often indistinctly, what they can discriminate.

The existence of such dissociations - attention without consciousness and consciousness without attention - should not be surprising when considering their different functions. Attention is a set of mechanisms whereby the brain selects a subset of the incoming sensory information for higher level processing, while the nonattended portions of the input are analyzed at a lower band width. For example, in primates, about one million fibers leave each eye and carry on the order of one megabyte per second of raw information. One way to deal with this deluge of data is to select a small fraction and process this reduced input in real time, while the nonattended data suffer from benign neglect. Attention can be directed by bottom-up, exogenous cues or by top-down endogenous features and can be applied to a spatially restricted part of the image (focal, spotlight of attention), an attribute (e.g., all red objects), or to an entire object. By contrast, consciousness appears to be involved in providing a kind of "executive summary" of the current situation that is useful for decision making, planning, and learning (Baars).

\section{Changes in the Level of Consciousness}

In neurology, a distinction is traditionally made between the level of consciousness and the content of consciousness. When you fall asleep, for example, the level of consciousness decreases to the point that you become virtually unconscious - the degree to which you are conscious (of anything) becomes progressively less and less. The content of consciousness, instead, refers to the particular experience you're having at any given time, for the same level of consciousness. It is useful to maintain this distinction when considering the 
NCG, and begin by considering changes in the level of consciousness.

\section{Sleep}

Sleep offers a commonplace, daily demonstration that the level of consciousness can change dramatically. In the laboratory, a subject is awakened during different stages of sleep and asked to report "anything that was going through your mind just before waking up." What is most noteworthy for the present purposes is that a number of awakenings from non-REM (NREM) sleep, especially early in the night when EEG slow waves are prevalent, can yield no report whatsoever. Thus, early slow-wave sleep is the only phase of adult life during which healthy human subjects may deny that they were experiencing anything at all. When present, reports from NREM sleep early in the night are often short and thought-like. However, especially later in the night, reports from NREM sleep can be longer, more hallucinatory and, generally speaking, more dream-like. On the other hand, awakenings during REM sleep almost always yield dreams - vivid conscious experiences, sometimes organized within a complex narrative structure (Hobson et al. 2000; Hobson \& Pace-Schott 2002).

What are the processes underlying the fading of the level of consciousness during early slow-wave sleep? Though metabolic rates decrease in many cortical areas, especially frontal and parietal areas, the thalamocortical system is far from shutting down. Instead, triggered by a decrease in acetylcholine and other modulators, cortical and thalamic neurons undergo slow oscillations $(1 \mathrm{~Hz}$ or less) between an up and a down state (Steriade et al. 2001). During the up state, cortical cells remain depolarized at waking levels for around a second and fire at waking rates, often in the gamma range (Destexhe et al. 2007). However, the up state of NREM sleep is not stable as in wakefulness and REM sleep, but it is inherently bistable. The longer neurons remain depolarized, the more likely they become to precipitate into a hyperpolarized down state - a complete cessation of synaptic activity that can last for a tenth of a second or more - after which they revert to another up state. The transition from up to down states appears to be due to depolarization-dependent potassium currents and to short-term synaptic depression, both of which increase with the amount of prior activation (Steriade 2003). The slow oscillation is found in virtually every cortical neuron and is synchronized across the cortical mantle by cortico-cortical connections, which is why the EEG records high-voltage, low-frequency waves.
An intriguing possibility is that changes in the level of consciousness during sleep may be related to the degree of bistability of thalamocortical networks (Tononi 2004b; Massimini et al. 2007). In a recent study, transcranial magnetic stimulation (TMS) was used in conjunction with high-density EEG to record cortical evoked responses to direct stimulation in waking and sleep (Massimini et al. 2005; Massimini et al. 2007). During wakefulness, TMS induced a sustained response made of rapidly changing patterns of activity that persisted until $300 \mathrm{~ms}$ and involved the sequential activation of specific brain areas, depending on the precise site of stimulation. During early NREM sleep, however, the brain response to TMS changed markedly. When applied to lateral cortical regions, the activity evoked by TMS remained localized to the site of stimulation, without activating connected brain regions, and lasted for less than $150 \mathrm{~ms}$ (Massimini et al. 2005). This finding indicates that during early NREM sleep, when the level of consciousness is reduced, effective connectivity among cortical regions breaks down, implying a corresponding breakdown of cortical integration. Computer simulations suggest that this breakdown of effective connectivity may be due to the induction of a local down state (Esser \& Tononi, in preparation). When applied over centromedian parietal regions, each TMS pulse triggered a stereotypical response implying the induction of a global down state: a full-fledged, high-amplitude slow wave (Massimini et al. 2007) that closely resembled spontaneous ones and that traveled through much of the cortex (Massimini et al. 2004). Such stereotypical responses could be induced even when, for the preceding seconds, there were no slow waves in the spontaneous EEG, indicating that perturbations can reveal the potential bistability of a system irrespective of its observed state. Altogether, these TMS-EEG measurements suggest that the sleeping brain, despite being active and reactive, becomes inherently bistable: it either breaks down in causally independent modules or bursts into a global, stereotypical response. By contrast, during REM sleep late in the night, when dreams become long and vivid and consciousness returns to levels close to those of wakefulness, the responses to TMS also recover and come to resemble more closely those observed during wakefulness: evoked patterns of activity become more complex and spatially differentiated, although some late components are still missing (Massimini \& Tononi, unpublished).

\section{Anesthesia}

The most common among exogenous manipulations of the level of consciousness is general 
anesthesia. Anesthetics come in two main classes: intravenous agents used for induction, such as propofol and ketamine, which are generally administered together with sedatives such as midazolam and dexmedetomidine; and inhaled agents, such as isoflurane, sevoflurane, and desflurane or the gases xenon and nitrous oxide. The doses of inhaled anesthetics are usually referred to their minimum alveolar concentration (MAC): a MAC value of 1 is the dose that prevents movement in 50\% of subjects in response to a painful surgical stimulation. At low MAC values $(0.1-0.2)$ anesthetics produce amnesia, first explicit and then implicit. Frequently there are distortions of time perception, such as slowing down and fragmentation, and a feeling of disconnection from the environment. Also, at low MAC values anesthetics produce increasing sleepiness and make arousal progressively more difficult, suggesting that to some extent they can mimic neurophysiological events underlying sleep. At around 0.3 MAC people experience a decrease in the level of consciousness, also described as a "shrinking" in the field of consciousness, as if they were kept on the verge of falling asleep. MAC-awake, usually around $0.3-0.4 \mathrm{MAC}$, is the point at which response to verbal command is lost in 50\% of patients and is considered the point at which consciousness is lost (LOC). The transition to unconsciousness (LOC) appears to be rather brusque, not unlike the collapse of muscle tone that usually accompanies it, suggesting that neural processes underlying consciousness change in a nonlinear manner. At concentrations above LOC, movements are still possible, especially partially coordinated responses to painful stimuli, suggesting that some degree of "unconscious" processing is still possible. Complete unresponsiveness is usually obtained just above MAC of 1.0 .

At the cellular level, many anesthetics have mixed effects, but the overall result is a decrease in neuronal excitability by either increasing inhibition or decreasing excitation. Most anesthetics act by enhancing GABA inhibition or by hyperpolarizing cells through an increase of potassium-leak currents. They can also interfere with glutamatergic transmission and antagonize acetylcholine at nicotinic receptors (Campagna et al. 2003; Franks 2006). But what are critical circuits mediating the LOC induced by anesthetics?

A considerable number of neuroimaging studies in humans have recently shed some light on this issue (Alkire \& Miller 2005). The most consistent effects produced by most anesthetics at LOC is a reduction of thalamic metabolism and blood flow, suggesting the possibility that the thalamus may serve as a consciousness switch (Alkire et al. 2000). (Another common site of action of several anesthetics is the posterior cingulate/medial parietal cortical areas, as well as the medial basal forebrain). However, both PET and fMRI signals mostly reflect synaptic activity rather than cellular firing, and the thalamus receives a massive innervation from cortex. Moreover, the relative reduction in thalamic activity occurs on a background of a marked decrease in global metabolism (30-60\%) that involves many cortical regions. Thus, thalamic activity as recorded by neuroimaging may represent an especially sensitive, localized readout of the extent of widespread cortical deactivation, rather than the final common pathway of unconsciousness (Ori et al. 1986). In fact, spontaneous thalamic firing in animal models of anesthesia is mostly driven by corticothalamic feedback (Vahle-Hinz et al. 2007), and the metabolic effects of enflurane on the thalamus can be abolished by an ipsilateral cortical ablation (Nakakimura et al. 1988), suggesting that the switch in thalamic unit activity is driven primarily through a reduction in afferent corticothalamic feedback more than by a direct effect of anesthesia on thalamic neurons. Recently, thalamic activity was recorded using depth electrodes in a patient undergoing anesthesia for the implant of a deep brain stimulator (Velly et al. 2007). With either propofol or sevoflurane, when the patient lost consciousness, the cortical EEG changed dramatically. However, there was little change in the thalamic EEG until almost 10 min later. This result implies that the deactivation of cortex alone is sufficient for loss of consciousness, and conversely that thalamic activity alone is insufficient to maintain it.

These findings do not imply that the thalamus is irrelevant, but rather suggest that its effects on consciousness are largely accounted for by its effects on cortical circuits, most notably by providing a tonic facilitatory action that can help to restore consciousness, or cause its collapse when it fails. A dramatic demonstration is provided by a recent study, where rats kept under anesthetic concentration of sevoflurane could be awakened by a minute injection of nicotinic agents in an intralaminar thalamic nucleus (Alkire et al. 2007). Conversely, GABA agonists infused into the same side caused an immediate loss of consciousness (Miller et al. 1989; Miller \& Ferrendelli 1990) (though nicotinic antagonists did not).

\section{Coma and Vegetative States}

While consciousness may nearly fade during certain phases of sleep and be kept at very low levels for a prescribed period during general anesthesia, coma and vegetative states are characterized by a loss of consciousness that is hard or impossible to 
reverse. Coma - an enduring sleep-like state of immobility with eyes closed from which the patient cannot be aroused - represents the paradigmatic form of pathological loss of consciousness. Typically, coma is caused by a suppression of thalamocortical function by drugs, toxins, or internal metabolic derangements. Other causes of coma are head trauma, strokes, or hypoxia due to heart failure, which again cause widespread destruction of thalamocortical circuits. Smaller lesions of the reticular activating system can also cause unconsciousness, presumably by deactivating the thalamocortical system indirectly.

Patients who survive a coma may recover, while others enter the so-called vegetative state, in which eyes reopen, giving the appearance of wakefulness, but unresponsiveness persists. Soon, regular sleep/waking cycles follow. Respiration, other autonomic functions and brainstem functions are relatively preserved, and stereotypic, reflex-like responses can occur, including yawning and grunting, but no purposeful behavior. Patients may remain vegetative for a long time or may emerge to a "minimally conscious state." This is distinguished from the vegetative state by the occasional occurrence of some behaviors that do not appear to be purely reflexive, suggesting that some degree of consciousness may be present.

Post-mortem analysis in vegetative patients reveals that the brainstem and hypothalamus, and specifically the reticular activating system, are largely spared, which explains why patients look awake. Usually the vegetative state is due to widespread lesions of gray matter in neocortex and thalamus, to widespread white-matter damage and diffuse axonal injury, or to bilateral thalamic lesions, especially of the paramedian thalamic nuclei. It is not clear whether thalamic damage is largely a reflection of diffuse cortical damage (as in metabolic studies of anesthesia, changes in the thalamus are much more concentrated and therefore easier to document than changes in cortex) or whether even isolated thalamic damage can cause persistent unconsciousness. Matrix, calbindin-positive thalamic cells, which are most numerous within intralaminar nuclei, project diffusely to supragranular layers of cortex (Jones 1998) where they facilitate coherent highfrequency oscillations (Joliot et al. 1994). Such cells may be especially important in enabling effective interactions among many cortical regions: if their activating function is lost, patients may remain vegetative even though cortical tissue may be relatively intact. Indeed, Steven Laureys and colleagues showed that recovery from a vegetative state was associated with the restoration of functional connectivity between intralaminar thalamic nuclei and prefrontal and anterior cingulate cortices (Laureys et al. 2000a). A recent study by Niko Schiff and colleagues shows the role of the thalamus even more dramatically: bilateral deep brain electrical stimulation of the central thalamus restored a degree of behavioral responsiveness in a patient who had remained in a minimally conscious state for 6 years following brain trauma (Schiff et al. 2007).

In vegetative states, brain metabolism is globally reduced by 50 to $60 \%$, most notably in regions such as the posterior cingulate cortex and the precuneus (Laureys et al. 2004; Schiff 2006a). These are also the areas that reactivate most reliably if a patient regains consciousness. A recent case study reported an extraordinary recovery of verbal communication and motor function in a patient who had remained in a minimally conscious state for 19 years (Voss et al. 2006). Diffusion tensor MRI showed increased fractional anisotropy (assumed to reflect myelinated fiber density) in posteromedial cortices, encompassing cuneus and precuneus. These same areas showed increased glucose metabolism as studied by PET scanning, likely reflecting the neuronal regrowth paralleling the patient's clinical recovery. This and other neuroimaging studies of vegetative or minimally conscious patients are demonstrating ever more clearly that a purely clinical diagnosis of persistent loss of consciousness may at times be dramatically inaccurate. It was already known that patients with locked-in syndromes due to pontine lesions that left them almost completely paralyzed may seem unresponsive, but if they preserve even a minimal ability to signal (typically by moving an eye or eyelid up or down) they can communicate a rich subjective experience. What about patients who show no voluntary movement at all? Several imaging studies have now shown that, even in completely unresponsive patients, as long as significant portions of the thalamocortical system are preserved, cognitive stimuli can induce patterns of activation similar to those seen in healthy subjects (Laureys et al. 2004; Schiff 2006b). As we have seen in a previous section, stimuli that are not perceived consciously can still activate appropriate brain areas. Thus, inferring the presence of consciousness may be unwarranted. However, in a recent, thoughtprovoking study, a clinically vegetative, seemingly unresponsive patient was put in the scanner and asked to imagine playing tennis or navigating through her room. Remarkably, the patient showed fMRI activation patterns of the appropriate cortical regions, exactly like healthy subjects. Obviously, these activations could not be due to unconscious processing of stimuli (Owen et al. 2006). Of note, this patient had widespread frontal lesions, while posterior cortex was largely preserved. 


\section{Seizures}

The abnormal, hypersynchronous discharge of neurons is a frequent cause of short-lasting impairments of consciousness. Consciousness is lost or severely affected in so-called generalized seizures, such as absence and tonic-clonic seizures, and to a lesser extent in complex partial seizures. Absence seizures, which are more frequent in children, are momentary lapses of consciousness during which a child stops what she was doing and stares straight ahead blankly. Absence seizures are accompanied by spike-and-wave complexes at around $3 \mathrm{~Hz}$ in the EEG, reflecting cycles of synchronous firing and silence of large numbers of neurons. There is great variability in the degree of unresponsiveness both across subjects and, within the same subject, between seizures. Sometimes, simple behaviors such as repetitive tapping or counting can proceed unimpaired during the seizures, but more complex tasks come to a halt.

Generalized convulsive seizures usually comprise a tonic phase of muscle stiffening, followed by a clonic phase with jerking of the arms and legs. After the convulsion the person may be lethargic or in a state of confusion for minutes up to hours. During the tonic phase of a convulsive seizure neural activity is greatly increased, as indicated by high frequency activity in the EEG. The clonic phase is accompanied by synchronous spikes and waves in the EEG, corresponding to millions of neurons alternately firing in strong bursts and turning silent. Loss of consciousness during the tonic phase of generalized seizures is noteworthy because it occurs at times when neuronal activity is extremely high and synchronous.

Partial complex seizures often begin with strange abdominal sensations, fears, premonitions, or automatic gestures. The person progressively loses contact with the environment, exhibits a fixed stare, and is unable to respond adequately to questions or commands. Stereotyped, automatic movements are common. Complex partial seizures usually last from $15 \mathrm{sec}$ to $3 \mathrm{~min}$. Seizure activity is usually localized to the medial temporal lobe.

The diagnosis of seizures is made through clinical observation and EEG recordings. In absence and tonic-clonic seizures the scalp EEG shows diffuse abnormalities, suggesting a generalized involvement of brain networks, whereas in partial complex seizures the abnormalities are confined to a medial temporal focus on one side. However, neuroimaging studies using SPECT, PET, and fMRI, and depth EEG recordings in humans and animals have revealed that generalized seizures do not affect all brain areas indiscriminately, whereas complex partial seizures alter brain ac- tivity less focally than initially thought (Blumenfeld \& Taylor 2003; Blumenfeld et al. 2003; Blumenfeld et al. 2004; Blumenfeld 2005). In fact, it now appears that all seizures causing an impairment of consciousness are associated with changes in activity in three sets of brain areas, namely: i) increased activity in the upper brainstem and medial thalamus; ii) decreased activity in the anterior and posterior cingulate, medial frontal cortex, and precuneus; iii) altered activity in the lateral and orbital frontal cortex and in the lateral parietal cortex. In tonic-clonic seizures, fronto-parietal association areas show increased activity, while the pattern is more complex and variable in absence seizures, though parietal areas are usually deactivated. Complex partial seizures show decreased activity in frontal and parietal association cortex, which is associated with the onset of slow waves similar to those of sleep or anesthesia (rather than to epileptiform discharges).

At this stage, it is not clear which of these three sets of areas, alone or in combination, are crucial for the loss of consciousness. However, two things are clear: first, the areas involved in the loss of consciousness associated with seizures correspond to those affected in sleep, anesthesia, and the vegetative state, pointing to a common substrate for the most common forms of loss of consciousness; and second, especially during the tonic phase of convulsive seizures, it would seem that consciousness is lost when neurons are excessively and synchronously active, rather than inactive.

\section{Changes in the Content of Consciousness-Localizing the NCC}

As we have seen, much can be learned by studying how brain activity changes with changes in the overall level of consciousness. A complementary approach is to examine how brain activity changes when a specific content of consciousness changes - for example, a visual stimulus becomes visible or invisible - while everything else, including the overall level of consciousness as well as the sensory input, remains as constant as possible. Recording spike trains from individual neurons or measuring hemodynamic signals while the subject is looking at a stimulus in an MR scanner allows researchers to investigate what kind of neuronal activity is correlated with the conscious perception of the stimulus rather than with the mere presentation of the stimulus. The goal is to follow the footprints of consciousness in the brain by ultimately identifying the neural correlates of consciousness (NCG) - the minimal neuronal mechanisms that are jointly sufficient for any one specific conscious percept (Crick \& Koch 1995, 
1998, 2003). Thus, every phenomenal, subjective state will have associated NCG: one for seeing a red patch, another one for seeing grandmother, yet a third one for hearing a siren, another one for feeling free to act one way or another and so on. Perturbing or inactivating the NCG for any one specific conscious experience will affect the percept or cause it to disappear. If the NCG could be induced artificially, for instance by cortical microstimulation in a prosthetic device or during neurosurgery, the subject will experience the associated percept. This definition of the NCG stresses the word "minimal," because the question of interest is which subcomponents of the brain are actually needed. For instance, while neural activity in the cerebellum may ultimately affect some behaviors elicited by the presentation of a stimulus (such as eye movements), it is likely that such activity has little to do with the generation of the conscious percept of that stimulus, and thus is not part of the NCG.

Many questions can be asked: What characterizes the NCC for a specific experience? What do the NCG for different experiences have in common? Do the NCG involve just the thalamocortical system, or do they extend to structures such as the hippocampus, the claustrum, or the basal ganglia? Do they involve all pyramidal neurons in cortex at any given point in time? Or only a subset of cells in frontal lobes that project to the sensory cortices in the back? Only layer 5 cortical cells? Neurons that fire in a phasic, synchronous, or oscillatory manner? Or neurons that are included in reentrant loops? These are some of the proposals that have been advanced over the past years (Chalmers 2000).

Much of the contemporary work aimed at characterizing the NCG has concentrated on changes in specific visual contents of consciousness (or awareness, which is here used interchangeably), in part because visual experience is easy to manipulate experimentally, and in part because more is known about the anatomy and function of visual areas in primates than about any other brain regions. Visual psychologists have perfected a number of techniques - masking, binocular rivalry, continuous flash suppression, motion-induced blindness, change blindness, inattentional blindnessin which the seemingly simple and unambiguous relationship between a physical stimulus in the world, the resulting neural activity, and its associated percept is disrupted (Kim \& Blake 2005). We first consider what such approaches have revealed about the brain areas that underlie the NCG, focusing specifically on the visual system. In the following sections we ask whether the NCG for visual percepts corresponds to some special kind of neural activity.

\section{A Test Case: The NCC in Visual Cortex}

Many experiments exploit perceptual illusions in which the physical stimulus remains fixed while the percept fluctuates. The best known example is the Necker cube, whose 12 lines can be perceived in one of two different ways in depth. Another perceptual illusion that is easy to vary parametrically is binocular rivalry (Blake \& Logothetis 2002): a small image, for example a horizontal grating, is presented to the left eye and another image, for example a vertical grating, is shown to the corresponding location in the right eye. In spite of the constant visual stimulus, observers consciously see the horizontal grating alternate every few seconds with the vertical one. The brain does not allow for the simultaneous perception of both images.

Macaque monkeys can be trained to report whether they see the left or the right image. The distribution of the switching times and the way in which changing the contrast in one eye affects the reports leaves little doubt that monkeys and humans experience the same basic phenomenon. In a series of elegant experiments, Logothetis and colleagues (Leopold \& Logothetis 1996; Logothetis 1998) recorded from a variety of visual cortical areas in the awake macaque monkey while the animal performed a binocular rivalry task. In primary visual cortex (V1), only a small fraction of cells weakly modulated their response as a function of the percept of the monkey. The majority of cells responded to one or the other retinal stimulus with little regard to what the animal perceived at the time. In contrast, in a highlevel cortical area such as the inferior temporal (IT) cortex along the ventral pathway, almost all neurons responded only to the perceptual dominant stimulus, that is, to the stimulus that was being reported. For example, when a face and a more abstract design were presented, one of these to each eye, a "face" cell fired only when the animal indicated by its performance that it saw the face and not the design presented to the other eye. This result implies that the NCG involve activity in neurons in inferior temporal cortex (but not, of course, that the NCG are local to IT).

In a related perceptual phenomenon, flash suppression (Wolfe 1984), the percept associated with an image projected into one eye is suppressed by flashing another image into the other eye (while the original image remains). A methodological advantage over binocular rivalry is that the timing of the perceptual transition is determined by an external trigger rather than by an internal event. The majority of responsive cells in inferior temporal cortex and in the superior temporal sulcus follow the monkey's behavior - and therefore its percept. That is, when the animal perceives a cell's preferred stimulus, the neuron fires; when the stimulus 
is present on the retina but is perceptually suppressed, the cell falls silent, even though legions of V1 neurons fire vigorously to the same stimulus (Sheinberg \& Logothetis 1997). Single neuron recordings in the medial temporal lobe of epileptic patients during flash suppression likewise demonstrate abolition of their responses when their preferred stimulus is present on the retina but not seen (Kreiman et al. 2002).

In a powerful combination of binocular rivalry and flash suppression, a stationary image in one eye can be suppressed for minutes on end by continuously flashing different images into the other eye (continuous flash suppression; Tsuchiya \& Koch 2005). This paradigm lends itself naturally to further investigation of the relationship between neural activity - whether assayed at the single neuron or at the brain voxel level - and conscious perception. Imaging experiments with such perceptual illusions demonstrate quite conclusively that BOLD activity in the upper stages of the ventral pathway (e.g., the fusiform face area and the parahippocampal place area) follow the percept and not simply the retinal stimulus (Tong et al. 1998; Rees \& Frith 2007).

There is a lively debate about the extent to which neurons in primary visual cortex simply encode the visual stimulus or are directly contributing to the subject's conscious percept (Tong 2003). That is, is V1 part of the NCG (Crick \& Koch 1995)? It is clear that retinal neurons are not part of the NCG for visual experiences. While retinal neurons often correlate with visual experience, the spiking activity of retinal ganglion cells does not accord with visual experience (e.g., there are no photoreceptors at the blind spot; yet no hole in the field of view is apparent, in dreams vivid imagery occurs despite closed eyes, and so on). A number of compelling observations link perception with fMRI BOLD activity in human V1 and even in the lateral geniculate nucleus (LGN) (Tong et al. 1998; Lee et al. 2005). These data are at odds with single-neuron recordings from the monkey. This discrepancy is probably explained by the often tight relationship between consciousness and attention that was mentioned above (see section "Consciousness and Attention"). Unless attentional effects are carefully controlled for, their neural correlates cannot be untangled from those of consciousness (Huk et al. 2001; Tse et al. 2005). This has now been achieved in an elegant study of Lee, Blake, and Heeger (2007). Using a dual-task paradigm they found that hemodynamic BOLD activity in human V1 reflects attentional processes but does not directly correlate with the conscious percept of the subject. The experiment by Bahrami and colleagues (Bahrami et al. 2007) comes to a similar conclusion - they show that the fMRI signal in V1 associated with percep- tually invisible line drawings can still be modulated by selective visual attention. Also, Haynes and Rees (2005) exploited multivariate decoding techniques to read out perceptually suppressed information (the orientation of a masked stimulus) from V1 BOLD activity, even though the stimulus orientation was so efficiently masked that subjects performed at chance levels when trying to guess the orientation. That is, although subjects did not give any behavioral indication that they saw the orientation of the stimulus, its slant could be predicted on a single-trial basis with better-thanchance odds from the V1 (but not from V2 or V3) BOLD signal.

In conclusion, information pertaining to visual stimulus can be registered in primary visual cortex that may or may not be accessible to the subject. It may be modulated by attention, but it does not appear to correlate directly with the subject's percept. In that sense much of the neural activity in $\mathrm{V} 1$ probably does not belong to the NCG. Moreover, as suggested by PET experiments in patients in a persistent vegetative state, in whom stimuli evoked strong but localized activity in primary auditory and primary somatosensory cortices (Laureys et al. 2000b; Laureys et al. 2002), it may well be that none of the primary sensory cortices contributes directly to sensory consciousness.

\section{Consciousness and Neural Dynamics}

The studies discussed above indicate that, even within the cerebral cortex, changes in neural activity do not necessarily correlate with changes in conscious experience. Also, we saw earlier that most of the cortex is active during early NREM sleep and anesthesia, not to mention during generalized seizures, but subjects have little conscious content to report. Thus, it is natural to suggest that some additional dynamic feature of neural activity must be present to generate conscious content. Here we consider the roles of i) sustained versus phasic activity, ii) reentrant versus feedforward activity, and iii) synchronous or oscillatory activity.

\section{Sustained versus Phasic Activity}

A plausible idea is that neural activity may contribute to consciousness only if it is sustained for a minimum period of time, perhaps around a few hundred ms. At the phenomenological level, there is no doubt that the "now" of experience unfolds at a time scale comprised between tens and several hundred ms (Bachmann 2000) and in some aspects may even stretch to one or two seconds (Poppel \& Artin 1988). Other experiments have made use of the attentional blink 
phenomenon: when an observer detects a target in a rapid stream of visual stimuli, there is a brief period of time during which the detection of subsequent targets is impaired. Remarkably, targets that directly follow the first target are less impaired than those that follow after 200 to $400 \mathrm{~ms}$ (Raymond et al. 1992). By manipulating attention, identical visual stimuli can be made conscious or unconscious. In such studies, event-related potentials reflecting early sensory processing (the $\mathrm{Pl}$ and $\mathrm{Nl}$ components) were identical for seen and unseen stimuli but quickly diverged around $270 \mathrm{~ms}$, suggesting that stimuli only become visible when a sustained wave of activation spreads through a distributed network of cortical association areas (Vogel et al. 1998; Sergent et al. 2005).

More generally, the requirement for sustained discharge might account for why fast, reflex responses of the kind mediated by, say, the spinal cord, do not seem to contribute to consciousness. Fast, reflex-like responses also take place in the cerebral cortex. For example, the action-oriented dorsal visual stream rapidly adjusts movements that remain outside of consciousness (Goodale \& Milner 2005). Perhaps these reflexlike adjustments to fast-changing aspects of the environment that must be tracked online are incompatible with the development of sustained discharge patterns. By contrast, such sustained patterns may be necessary for the ventral stream to build a stable representation of a visual scene. By the same token, if the ventral stream could be forced to behave in a reflex-like manner, it should cease to be part of the NCG. Indeed, this can be achieved by pushing the ventral system to perform ultra-rapid categorizations such as deciding whether a natural image contains an animal or not (Thorpe et al. 1996; Bacon-Mace et al. 2005; Kirchner \& Thorpe 2006). In such cases, a sweep of activity travels from the retina through several stages of feedforward connections along the hierarchy of ventral visual areas, until it elicits an appropriate categorization response. This process takes as little as $150 \mathrm{~ms}$, which leaves only about $10 \mathrm{~ms}$ of processing per stage. Thus, only a few spikes can be fired before the next stage produces its output, yet they are sufficient to specify selective responses for orientation, motion, depth, color, shape, and even animals, faces, or places conveying most of the relevant information about the stimulus (Hung et al. 2005). While this fast feedforward sweep within the ventral system is sufficient for the near-automatic categorization of stimuli and a behavioral response, it seems insufficient to generate a conscious percept (VanRullen \& Koch 2003). For example, if another image (the mask) is flashed soon after the target image, subjects are still able to categorize the target, though they may deny having seen it consciously. Thus, consciousness would seem to require that neural activity in appropriate brain structures lasts for a minimum amount of time, perhaps as much as is needed to guarantee interactions among multiple areas.

On the other hand, other data would seem to suggest that it may actually be the phasic, onset, or offset discharge of neurons that correlates with experience. The most stringent data come again from studies of visual masking (for a review see Macknik 2006). To be effective, masking stimuli must either precede (forward masking) or follow (backward masking) target stimuli at appropriate time intervals and usually need to be spatially contiguous. Macknik, MartinezConde, and collaborators showed, using a combination of psychophysics, unit recordings in animals, and neuroimaging in humans, that for simple, unattended target stimuli, masking stimuli suppress visibility if their "spatiotemporal edges" overlap with the spatiotemporal edges of the targets, that is, if they begin or end when target stimuli begin or end, in space and time. They confirmed that such spatiotemporal edges correspond to transient bursts of spikes in primary visual cortex. If these bursts are inhibited, for example if the offset discharge elicited by the target stimulus is obliterated by the onset of the mask, the target becomes invisible. Most likely, these spatiotemporal edges of increased firing are both generated (target stimuli) and suppressed (masking stimuli) by mechanisms of lateral inhibition, which are ubiquitous in sensory systems (Macknik 2006).

Additional evidence for the importance of phasic, transient activation of neurons in determining the visibility of stimuli comes from studies of microsaccadesthe small, involuntary movements that our eyes make continually (Martinez-Conde et al. 2004). If microsaccades are counteracted by image stabilization on the retina, stationary objects fade and become completely invisible. In a recent study (Martinez-Conde et al. 2006), subjects were asked to fixate a central dot (which tends to reduce microsaccades) while attending to a surrounding circle. Soon, the circle fades and merges into the background (the Troxler illusion). It was found that before a fading period, the probability, rate, and magnitude of microsaccades decreased. Before transitions toward visibility, the probability, rate, and magnitude of microsaccades increased, compatible with the hypothesis that microsaccades are indeed necessary for visibility. Importantly, in macaque monkeys, when an optimally oriented line was centered over the receptive field of cells in V1, the cells' activity increased after microsaccades and tended to emit bursts 
(Martinez-Conde et al. 2002), suggesting again that phasic activity may be crucial for visibility.

One should remember, however, that the importance of phasic discharges for stimulus awareness has only been demonstrated for early visual cortex; but, as we saw above, the NCC are more likely to lie elsewhere. Psychophysically, masking is similarly effective when masking stimuli are presented monoptically (through the same eye as the target) and dichoptically (through the other eye). The analysis of fMRI data in humans shows that a correlate of monoptic visual masking can be found in all retinotopic visual areas, whereas dichoptic masking is only seen in retinotopic areas downstream of V2 within the occipital lobe (Tse et al. 2005), suggesting an anatomical lower bound for the NCC. Thus, it could be that phasic onset and offset discharges are important not in and of themselves, but because they are particularly effective in activating downstream areas that directly support the NCC. In these downstream areas, perhaps, the NCG may actually require sustained firing. Indeed, the duration of the activation of face-selective neurons in IT is strongly correlated with the visibility of masked faces (Rolls et al. 1999).

\section{Reentrant versus Feedforward Activity}

Another possibility is that it is not so much sustained firing that triggers the awareness of a stimulus, but rather the occurrence of a "reentrant" wave of activity (also described as recurrent, recursive, or reverberant) from higher to lower cortical areas. In this view, when a stimulus evokes a feedforward sweep of activity, it is not seen consciously, but it becomes so when the feedforward sweep is joined by a reentrant sweep (Lamme \& Roelfsema 2000). This logic could apply both to early sensory areas such as V1 and to higher areas such as IT. For example, when face neurons in the fusiform face area are first activated, we would not see a face consciously, although we could turn our eyes toward it or press a button to indicate our unconscious categorization as a face. However, when face-selective neurons receive a backward volley from some higher area, for example frontal cortex, the face would become visible.

This view is based on several considerations. An important one, though rarely confessed, is that a mere sequence of feedforward processing steps seems far too "straightforward" and mechanical to offer a substrate for subjective experience. Reentrant processes, by "closing the loop" between past and present activity, or between predicted and actual versions of the input, would seem to provide a more fertile substrate for giving rise to reverberations (Lorente de Nó 1938), generating emergent properties through cell assemblies
(Hebb 1949), implementing hypothesis testing through resonances (Grossberg 1999), linking present with past (Edelman \& Mountcastle 1978; Edelman 1989), and subject with object (Damasio 1999).

A more concrete reason why reentrant activity is an attractive candidate for the NCG is that it travels through back connections, of which the cerebral cortex is extraordinarily rich (Felleman \& Van Essen 1991). In primates, feedforward connections originate mainly in supragranular layers and terminate in layer 4. Feedback connections instead originate in both superficial and deep layers and usually terminate outside of layer 4. The sheer abundance of back connections in sensory regions suggests that they ought to serve some important purpose, and giving rise to a conscious percept might just fit the bill. However, back connections and associated reentrant volleys are just as numerous between $\mathrm{V} 1$ and visual thalamus, which is usually denied any direct contribution to awareness. Also, there does not seem to be any lack of back connections within the dorsal stream. It should be emphasized that the strength and termination pattern of back connections seem more suited to a modulatory/synchronizing role than to driving their target neurons. For example, the focal inactivation of area 18 can slightly increase or decrease discharge rates of units in area 17 , but it does not change their feature selectivity for location and orientation (Martinez-Conde et al. 1999). Also, the numerosity of backward connections is a natural consequence of the hierarchical organization of feedforward ones. For instance, cells in the LGN are not oriented, while cells in area 17 are. To be unbiased, feedback to any one LGN cell should come from area 17 cells of all orientations, which requires many connections; on the other hand, since at any given time only area 17 cells corresponding to a given orientation would be active, feedback effects would not be strong. If they were, properties of area 17 cells, such as orientation selectivity, would be transferred upon LGN cells, which they are not (to a first approximation). Backward signals certainly play a role in sensory function: for instance, they can mediate some extraclassical receptive field effects, provide a natural substrate for both attentional modulation and imagery, and can perhaps dynamically route feedforward processing according to prior expectations. But are backward signals really critical for consciousness?

The most intriguing data in support of a role for reentrant activity in conscious perception have come from neurophysiological experiments. In awake monkeys trained to signal whether or not they see a salient figure on a background, the early, feedforward response of V1 neurons was the same, no matter whether or not 
the monkey saw the figure (Super et al. 2001). However, a later response component was suppressed when the monkey did not see the figure. Light anesthesia also eliminated this later component without affecting the initial response. Late components are thought to reflect reentrant volleys from higher areas, although other studies have disputed this claim (Rossi et al. 2001). The late response component crucial for the visibility of stimuli under backward masking might also be due to a reentrant volley (Lamme \& Roelfsema 2000). In this case the timing of maximal backward masking should be independent of target duration, since it would be determined exclusively by the time needed for the early component to travel to higher areas and return to primary visual cortex. Instead, the timing of maximal masking depends on the timing of target offset, suggesting that the component that is obliterated is a feedforward offset discharge, not a reentrant one (Macknik 2006). Moreover, the late component can be dissociated from a behavioral response simply by raising the decision criterion (Super et al. 2001), and it can occur in the absence of report during change and inattentional blindness (Scholte et al. 2006). Perhaps in such cases subjective experience is present but, due to an insufficient involvement of frontal areas, it cannot be reported (Block 2005; Tsuchiya \& Koch 2005; Lamme 2006). But then, by the same token, why should we rule out subjective experience during the feedforward sweep?

Another main source of evidence for a role of reentrant volleys in consciousness comes from experiments using TMS. In an early experiment, Pascual-Leone and Walsh applied TMS to V5 to elicit large, moving phosphenes (Pascual-Leone \& Walsh 2001). They then applied another, subthreshold TMS pulse to a corresponding location in V1. When TMS to V1 was delivered after TMS to V5 ( +5 to $+45 \mathrm{~ms})$, subjects often did not see the V5 phosphene, and when they saw one, it was not moving. Their interpretation was that disruption of activity in $\mathrm{V} 1$ at the time of arrival of a reentrant volley from $\mathrm{V} 5$ interferes with the experience of attributes encoded by V5. In a subsequent study (Silvanto et al. 2005), it was shown that, when a subthreshold pulse was applied over V5, followed 10 - $40 \mathrm{~ms}$ later by a suprathreshold pulse over V1, subjects reported a V5-like phosphene (large and moving), rather than a V1 phosphene (small and stationary). Their interpretation was that activity in V5 that, on its own, is insufficient to induce a moving percept can produce such a percept if the level of induced activity in $\mathrm{V} 1$ is high enough.

In another study (Boyer et al. 2005), subjects where shown either an oriented bar or a colored patch- stimuli that are processed in visual cortex. If, around $100 \mathrm{~ms}$ later, a TMS pulse was applied to V1, the stimulus became perceptually invisible, although on forced choice subjects could still discriminate orientation or color. This result indicates that, without any overt participation of $\mathrm{Vl}$, stimuli can reach extrastriate areas without eliciting a conscious percept, just as in blindsight patients. It also suggests that, since the forward sweep reaches $\mathrm{V} 1$ after just 30 or $40 \mathrm{~ms}$, the TMS pulse may abolish the awareness of the stimulus not so much by blocking feedforward transmission as by interfering with the backward volley (see also Ro et al. 2003). On the other hand, it cannot be ruled out that the TMS pulse may act instead by triggering a corticalthalamo-cortical volley that interferes with the offset discharge triggered by the stimulus, as may indeed be the case in backward masking.

Yet another study, this time using fMRI, examined the neural correlates of brightness (perceptual lightness) using backward masking. The psychometric visibility function was not correlated with the stimulated portion of V1, but with downstream visual regions, including fusiform cortex, parietal-functional areas, and with the sectors of $\mathrm{V} 1$ responding to the unstimulated surround (Haynes et al. 2005). Remarkably, visibility was also correlated with the amount of coupling (effective connectivity) between fusiform cortex and the portion of $\mathrm{V} 1$ that responded to the surround. Once again, this result could be explained by the activation of reentrant connections, though fMRI cannot distinguish between forward and backward influences.

\section{Synchronization and Oscillations}

Another influential idea is that consciousness may require the synchronization, at a fine temporal scale, of large populations of neurons distributed over many cortical areas, in particular via rhythmic discharges in the gamma range $(30-70 \mathrm{~Hz}$ and beyond) (Crick \& Koch 1990). The emphasis on synchrony ties in well with the common assumption that consciousness requires the "binding" together of a multitude of attributes within a single experience, as when we see a rich visual scene containing multiple objects and attributes that is nevertheless perceived as a unified whole (Singer 1999). According to this view, the NCG of such an experience would include two aspects: first, the underlying activation pattern (groups of neurons that have increased their firing rates) would be widely distributed across different areas of the cerebral cortex, each specialized in signaling a different object or attribute within the scene; and second, the firing of these activated neurons would be synchronized on a fast time scale to signal their binding into a single 
percept. In this respect, synchrony seems ideally suited to signal relatedness: for example, in the presence of a red square, some neurons would respond to the presence of a square, while others would respond to the presence of the color red. If they synchronize at a fast time scale, they would indicate to other groups of neurons that there is a red square, "binding" the two features together (Tononi et al. 1992). By contrast, signaling relatedness by increased firing rates alone would be more cumbersome and probably slower (Singer 1999). Also, fine-temporal-scale synchrony has the welcome property of disambiguating among multiple objectsif a green cross were present simultaneously with the red square, cross and green neurons would also be active, but false conjunctions would be avoided by precise phase locking (Tononi et al. 1992). Moreover, computational models predict that, for the same level of firing, synchronous input is more effective on target neurons than asynchronous input (Abeles 1991; Tononi et al. 1992), and indeed synchrony makes a difference to the outputs to the rest of the brain (Brecht et al. 1999; Schoffelen et al. 2005). Finally, large-scale models predict that synchrony in the gamma range occurs due to the reciprocal connectivity and loops within the thalamocortical system (Lumer et al. 1997a, b), and indeed phase alignment between distant groups of neurons in the gamma range anticipates by a few $\mathrm{ms}$ an increase in gamma-band power (Womelsdorf et al. 2007). In this respect, oscillatory activity, even when subthreshold, could further facilitate synchronous interactions by biasing neurons to discharge within the same time frame (Engel et al. 2001).

Experimental evidence concerning the role of synchrony/synchronous oscillations in perceptual operations was initially obtained in primary visual areas of anesthetized animals (for a review see Singer \& Gray 1995). For example, in primary visual cortex, neurons have been found that respond to a coherent object by synchronizing their firing in the gamma range. Stimulus-specific, gamma-range synchronization is greatly facilitated when the EEG is activated by stimulating the mesencephalic reticular formation (Munk et al. 1996; Herculano-Houzel et al. 1999). It is similarly facilitated by attention (Roelfsema et al. 1997) and by increases in a dominant stimulus under binocular rivalry even though firing rates may not change (Fries et al. 1997, 2001).

Other evidence has come from EEG studies of phase locking in humans. A recent study compared the neural correlates of words that were consciously perceived with those of masked words that had been processed and semantically decoded but had remained unconscious (Melloni et al. 2007). The results showed that consciously perceived words induced theta oscillations in multiple cortical regions until the test stimulus was presented and a decision reached, while a burst of gamma activity occurred over central and frontal leads just prior to and during the presentation of the test stimulus. Importantly, the earliest event distinguishing conscious and unconscious processing was not visible in the power changes of oscillations but in their phase locking. About $180 \mathrm{~ms}$ after presentation of stimuli that were consciously perceived, induced gamma oscillations recorded from a large number of regions exhibited precise phase locking both within and across hemispheres for around $100 \mathrm{~ms}$. These results have been interpreted to indicate that a transient event of gamma synchrony resets multiple parallel processes to a common time frame. The global theta rhythm that follows after this trigger event could provide the time frame for allowing a global integration of information provided by sensory inputs and internal sources. Or perhaps beta-gamma synchrony could enable the integration of activity patterns within local cortical areas, while theta synchrony would permit the integration of more globally distributed patterns: the more global the representation, the longer the time scale for the integration of distributed information.

It would be premature to conclude, however, that synchrony in one or another frequency band is necessarily a marker of NCG. As revealed by an increasing number of EEG, MEG, electrocorticography, and multiunit recordings, cognitive tasks are associated with complex modes of synchronous coupling among populations of neurons that shift rapidly both within and across different frequency bands, within and between areas, and in relation to the timing of the task (Womelsdorf et al. 2007). It would rather seem that, given the brain's remarkable connectivity, synchrony is an inevitable accompaniment of neural activity, and that it is bound to change just as activity patterns change, depending on the precise experimental conditions. Whether a particular kind of synchrony, for example in the gamma range, is uniquely associated with consciousness is still unclear. For example, high beta and gamma synchrony can be found in virtually every brain region investigated, including some that are unlikely to contribute directly to consciousness. Also, it is not yet clear whether synchrony in the gamma range vanishes during early NREM sleep, during anesthesia, or even during seizures. Human studies are still inconsistent on this point, whereas animal studies show a paradoxical increase in gamma synchrony when rats lose the righting reflex (thought to correspond more or less to the loss of consciousness in humans; see Imas et al. $2005 \mathrm{a}, \mathrm{b})$. It would seem that there can be synchrony 
without consciousness, though perhaps not consciousness without synchrony, at least in mammalian brains.

Finally, there are some difficult conceptual problems in characterizing synchrony as an essential ingredient that unifies perceptual states and thereby makes them conscious. While many experiences do indeed involve several different elements and attributes that are "bound" together into a unified percept, there are many other experiences, equally conscious, that do not seem to require much binding at all: for instance, an experience of pure darkness or of pure blue, or a loud sound that briefly occupies consciousness and has no obvious internal structure, would merely seem to require the strong activation of the relevant neurons, with no need for signaling relatedness to other elements, and thus no need for synchrony. Also, the idea that the NCG of a given conscious experience are given by active neurons bound by synchrony discounts the importance of inactive ones: information specifying that particular unified experience must be conveyed both by which neurons are active and which are not, yet for inactive neurons there does not seem to be anything to bind. On the other hand, if most neurons in the cortex were to become active hypersynchronously, as is the case in generalized seizures, they should result in maximal "binding," but consciousness vanishes rather than become more vivid..

\section{Consciousness from a Theoretical Perspective}

Progress in the study of the NCC will hopefully lead to a better understanding of what distinguishes neural structures or processes that are associated with consciousness from those that are not. But even if we come closer to this goal, we still need to understand why certain structures and processes have a privileged relationship with subjective experience. For example, why is it that neurons in thalamocortical circuits are essential for conscious experience, whereas cerebellar neurons, despite their huge numbers, are not? And what is wrong with many cortical circuits, including those in V1, that makes them unsuitable to yield subjective experience? Or why is it that consciousness wanes during slow-wave sleep early in the night, despite levels of neural firing in the thalamocortical system that are comparable to those in quiet wakefulness? Other questions are even more difficult to address in the absence of a theory. For example, is consciousness present in animals that have a nervous system considerably different from ours? And what about computerized robots or other artifacts that behave intelligently but are organized in a radically different way from human brains?

\section{Consciousness as Integrated Information}

To address these questions, it would seem that we need a theoretical approach that tries to establish, at the fundamental level, what consciousness is, how it can be measured, and what requisites a physical system must satisfy in order to generate it. The integrated information theory of consciousness represents such an approach (Tononi 2004b).

According to the theory, the most important property of consciousness is that is extraordinarily informative. This is because, whenever you experience a particular conscious state, it rules out a huge number of alternative experiences. Classically, the reduction of uncertainty among a number of alternatives constitutes information. For example, you lie in bed with eyes open and experience pure darkness and silence. This is one of the simplest experiences you might have, one that may not be thought as conveying much information. One should realize, however, that the informativeness of what you just experienced lies not in how complicated it is to describe, but in how many alternatives were ruled out when you experienced it: you could have experienced any one frame from any of innumerable movies, or the smoke and flames of your room burning, or any other possible scene, but you did not-instead, you experienced darkness and silence. This means that when you experienced darkness and silence, whether you think or not of what was ruled out (and you typically don't), you actually gained access to a large amount of information. This point is so simple that its importance has been overlooked.

It is essential to realize, however, that the information associated with the occurrence of a conscious state is integrated information. When you experience a particular conscious state, that conscious state is an integrated whole - it cannot be subdivided into components that are experienced independently. For example, the conscious experience of the particular phrase you are reading now cannot be experienced as subdivided into, say, the conscious experience of how the words look independently of the conscious experience of how they sound in your mind. Similarly, you cannot experience visual shapes independently of their color, or perceive the left half of the visual field of view independently of the right half.

Based on these and other considerations, the theory claims that the level of consciousness of a physical system is related to the repertoire of causal states (information) available to the system as a whole (integration). That is, whenever a system enters a particular state though causal interactions 
among its elements, it is conscious in proportion to how many system states it has thereby ruled out, provided these are states of the system as a whole, not decomposable into states of causally independent parts. More precisely, the theory introduces a measure of integrated information called $\Phi$, quantifying the reduction of uncertainty (i.e., the information) that is generated when a system enters a particular state through causal interactions among its parts, above and beyond the information that is generated independently within the parts themselves (hence integrated information). The parts should be chosen in such a way that they can account for as much nonintegrated (independent) information as possible.

If a system has a positive value of $\Phi$ (and it is not included within a larger subset having higher $\Phi$ ) it is called a complex. For a complex, and only for a complex, it is appropriate to say that when it enters a particular state, it generates an amount of integrated information corresponding to $\Phi$. Since integrated information can only be generated within a complex and not outside its boundaries, consciousness is necessarily subjective, private, and related to a single point of view or perspective (Tononi \& Edelman 1998). Some properties of complexes are worth pointing out. A given physical system, such as a brain, is likely to contain more than one complex, many small ones with low $\Phi$ values, and perhaps a few large ones. We suspect that in the brain there is at any given time a complex of comparatively much higher $\Phi$, which we call the main complex. Also, a complex can be causally connected to elements that are not part of it through ports-in and ports-out. In that case, elements that are part of the complex contribute to its conscious experience, while elements that are not part of it do not, even though they may be connected to it and exchange information with it through ports-in and ports-out. One should also note that the $\Phi$ value of a complex is dependent on both spatial and temporal scales that determine what counts as a state of the underlying system. In general, the relevant spatial and temporal scales are those that jointly maximize $\Phi$ (Tononi 2004b). In the case of the brain, the spatial elements and time scales that maximize $\Phi$ may be local collections of neurons such as minicolumns and periods of time comprised between tens and hundreds of milliseconds, respectively, though at this stage it is difficult to adjudicate between minicolumns and individual neurons.

\section{Accounting for Neurobiological Observations}

Measuring $\Phi$ and finding complexes is not easy for realistic systems, but it can be done for simple networks that bear some structural resemblance to different parts of the brain (Tononi 2004b, 2005). For example, by using computer simulations, it is possible to show that high $\Phi$ requires networks that conjoin functional specialization (due to its specialized connectivity, each element has a unique functional role within the network) with functional integration (there are many pathways for interactions among the elements.). In very rough terms, this kind of architecture is characteristic of the mammalian thalamo-cortical system: different parts of the cerebral cortex are specialized for different functions, yet a vast network of connections allows these parts to interact profusely. Indeed, the thalamo-cortical system is precisely the part of the brain which cannot be severely impaired without loss of consciousness.

Conversely, $\Phi$ is low for systems that are made up of small, quasi-independent modules. This may be why the cerebellum, despite its large number of neurons, does not contribute much to consciousness: its synaptic organization is such that individual patches of cerebellar cortex tend to be activated independently of one another, with little interaction between distant patches (Cohen \& Yarom 1998; Bower 2002).

Computer simulations also show that units along multiple, segregated incoming or outgoing pathways are not incorporated within the repertoire of the main complex. This may be why neural activity in afferent pathways (perhaps as far as V1), though crucial for triggering this or that conscious experience, does not contribute directly to conscious experience; nor does activity in efferent pathways (perhaps starting with primary motor cortex), though it is crucial for reporting each different experience.

The addition of many parallel cycles also generally does not change the composition of the main complex, although $\Phi$ values can be altered. Instead, cortical and subcortical cycles or loops implement specialized subroutines that are capable of influencing the states of the main thalamocortical complex without joining it. Such informationally insulated cortico-subcortical loops could constitute the neural substrates for many unconscious processes that can affect and be affected by conscious experience (Baars 1988; Tononi 2004a, b), such as those that enable object recognition, language parsing, or translating our vague intentions into the right words. At this stage, however, it is hard to say precisely which cortical circuits may be informationally insulated. Are primary sensory cortices organized like massive afferent pathways to a main complex "higher up" in the cortical hierarchy? Is much of prefrontal cortex organized like a massive efferent pathway? Do certain cortical areas, such as those belonging to the dorsal visual stream, remain partly segregated from 
the main complex? Do interactions within a corticothalamic minicolumn qualify as intrinsic miniloops that support the main complex without being part of it? Unfortunately, answering these questions and properly testing the predictions of the theory require a much better understanding of cortical neuroanatomy than is presently available (Ascoli 1999).

Other simulations show that the effects of cortical disconnections are readily captured in terms of integrated information (Tononi 2004b): a "callosal" cut produces, out of large complex corresponding to the connected thalamocortical system, two separate complexes, in line with many studies of split-brain patients (Gazzaniga 1995). However, because there is great redundancy between the two hemispheres, their $\Phi$ value is not greatly reduced compared to when they formed a single complex. Functional disconnections may also lead to a restriction of the neural substrate of consciousness, as is seen in neurological neglect phenomena, in psychiatric conversion and dissociative disorders, and possibly during dreaming and hypnosis. It is also likely that certain attentional phenomena may correspond to changes in the composition of the main complex underlying consciousness. Phenomena such as the attentional blink, where a fixed sensory input may at times make it to consciousness and at times not, may also be due to changes in functional connectivity: access to the main thalamocortical complex may be enabled or not based on dynamics intrinsic to the complex (Dehaene et al. 2003). Phenomena such as binocular rivalry may also be related, at least in part, to dynamic changes in the composition of the main thalamocortical complex caused by transient changes in functional connectivity (Lumer 1998). Computer simulations confirm that functional disconnection can reduce the size of a complex and reduce its capacity to integrate information (Tononi 2004b). While it is not easy to determine, at present, whether a particular group of neurons is excluded from the main complex because of hard-wired anatomical constraints, or is transiently disconnected due to functional changes, the set of elements underlying consciousness is not static, but form a dynamic complex or dynamic core (Tononi \& Edelman 1998).

From the perspective of integrated information, a reduction of consciousness during early sleep would be consistent with the ensuing bistability of cortical circuits. As we have seen, studies using TMS in conjunction with high-density EEG show that early NREM sleep is associated either with a breakdown of the effective connectivity among cortical areas, and thereby with a loss of integration (Massimini et al. 2005, 2007), or with a stereotypical global response suggestive of a loss of repertoire and thus of information (Massimini et al. 2007). Computer simulations also indicate that the capacity to integrate information is reduced if neural activity is extremely high and near synchronous, due to a dramatic decrease in the available degrees of freedom (Balduzzi and Tononi, in preparation). This reduction in degrees of freedom could be the reason why consciousness is reduced or eliminated in absence seizure and other conditions characterized by hypersynchronous neural activity.

Finally, we have seen that consciousness not only requires a neural substrate with appropriate anatomical structure and appropriate physiological parameters, it also needs time (Bachmann 2000). The theory predicts that the time requirement for the generation of conscious experience in the brain emerge directly from the time requirements for the build-up of an integrated repertoire among the elements of the thalamocortical main complex (Balduzzi and Tononi II, in preparation). To give an obvious example, if one were to perturb half of the elements of the main complex for less than a millisecond, no perturbations would produce any effect on the other half within this time window, and the repertoire measured by $\Phi$ would be equal to zero. After say $100 \mathrm{~ms}$, however, there is enough time for differential effects to be manifested, and $\Phi$ should grow.

\section{Some Implications}

The examples discussed above show that the integrated information theory can begin to account, in a coherent manner, for several puzzling facts about consciousness and the brain. This goes beyond proposing a provisional list of candidate brain areas for the NCG and of seemingly important neural ingredients, such as synchronization, sustained or phasic firing, reentrant activity, or widespread "broadcasting," without a principled explanation of why they would be important or whether they would be always necessary. Naturally, the integrated information theory converges with other neurobiological frameworks (e.g., Crick \& Koch 2003; Edelman 1989; Dehaene et al. 2006) and cognitive theories (Baars 1988) on certain key facts: that our own consciousness is generated by distributed thalamocortical networks, that reentrant interactions among multiple cortical regions are important, that the mechanisms of consciousness and attention overlap but are not the same, and that there are many "unconscious" neural systems.

The integrated information theory avoids the pitfalls associated with assigning conscious qualities to individual brain elements. For example, it is sometimes assumed loosely that the firing of specific thalamocortical elements (e.g., those for red) conveys some 
specific information (e.g., that there is something red) and that such information becomes conscious either as such, or perhaps if it is disseminated widely. However, a given thalamocortical element has no information about whether what made it fire was a particular color rather than a shape, a visual stimulus rather than a sound, a sensory stimulus rather than a thought. All it knows is whether it fired or not, just as each receiving element only knows whether it received an input or not. Thus, the information specifying "red" cannot possibly be in the message conveyed by the firing of any neural element, whether it is located in a high-order cortical area or whether it is broadcasting widely. According to the theory, that information resides instead in the reduction of uncertainty occurring when a whole complex enters one out of a large number of available states - the complex, and not its elements, is the locus of consciousness. Indeed, within a complex, both active and inactive neurons count, just as the sound of an orchestra is specified both by the instruments that are playing and by those that are silent. Though it would be too long to address the issue here, the theory proposes that, just as the quantity of consciousness is given by the amount of integrated information generated within a complex, the particular quality of consciousness - including the redness of red - is given by the specific informational relationships among the elements of the complex (Tononi 2004b).

The integrated information theory also predicts that consciousness depends exclusively on the ability of a system to integrate information, whether or not it has a strong sense of self, language, emotion, or is immersed in an environment, contrary to some common intuitions. Nevertheless, the theory recognizes that these same factors are important historically because they favor the development of neural circuits forming a main complex of high $\Phi$. For example, integrated information grows as that system incorporates statistical regularities from its environment and learns (Tononi et al. 1996). In this sense, the emergence of consciousness in biological systems is predicated on a long evolutionary history, on individual development, and on experiencedependent change in neural connectivity.

Finally, the integrated information theory says that the presence and extent of consciousness can be determined, in principle, also in cases in which we have no verbal report, such as in infants or animals, or in neurological conditions such as minimally conscious states, akinetic mutism, psychomotor seizures, and sleepwalking. In practice, of course, measuring $\Phi$ accurately in such systems will not be easy, but approximations and informed guesses are certainly conceivable. The theory also implies that consciousness is not an all-or-none property but is graded: specifically, it increases in proportion to a system's repertoire of available states. In fact, any physical system with some capacity for integrated information would have some degree of experience, irrespective of the stuff of which it is made, and independent of its ability to report.

At present, the validity of this theoretical framework and the plausibility of its implications rest on its ability to account, in a coherent manner, for some basic phenomenological observations and for some elementary but puzzling facts about consciousness and the brain. Experimental developments, such as ways to concurrently stimulate and record the activity of broad regions of the brain, should permit stringent tests of some of the theory's predictions. Especially important will be paradigms to test the counterintuitive prediction that experience can change even though the underlying neural activity stays the same: if one inactivates neurons that are silent, the current firing pattern would not change, but the repertoire of available neural states would shrink and consciousness should be diminished.

\section{Conclusions}

As this chapter demonstrates, the study of consciousness has entered an intense experimental phase, a refreshing change from the previous millennia when the only approach available to students of the mind was philosophical speculation. The two dominant experimental paradigms are the study of brain differences between the awake, conscious state and various degrees of unconsciousness, such as deep sleep, anesthesia, or coma, and the study of the brain basis of the changing content of consciousness, as in when one visual stimulus is consciously perceived but then becomes invisible and vice versa.

In order to make progress, it will be imperative to record from a large number of neurons simultaneously at many locations throughout the cortico-thalamic system and related satellites in behaving subjects. Such experiments cannot, of course, be done in humans. Progress in understanding the circuitry of consciousness therefore demands a battery of behaviors (akin to but different from the well-known Turing test for intelligence) that the subject - a newborn infant, immobilized patient, or nonhuman animal - has to pass before considering him, her, or it to possess some measure of conscious experience. This is not an insurmountable step for mammals such as the monkey or the mouse that share many behaviors and brain structures with humans. For example, one particular mouse model of 
contingency awareness (Han et al. 2003) is based on the differential requirement for awareness of trace versus delay associative eyeblink conditioning in humans (Clark \& Squire).

The growing ability of neuroscientists to manipulate in a reversible, transient, deliberate, and delicate manner identified populations of neurons using methods from molecular biology combined with optical stimulation (Aravanis et al. 2007; Han \& Boyden 2007; Zhang et al. 2007) enables the intrepid neuroengineer to move from correlation - observing that a particular conscious state is associated with some neural or hemodynamic activity - to causation. For example, rather than just noting that motion perception is associated with an elevated firing rate in projection neurons in cortical area MT, we will be able to perturb the system by inactivating genetically distinct subpopulations of cells in a highly targeted manner. Exploiting these increasingly powerful tools depends on the simultaneous development of appropriate behavioral assays and model organisms amenable to large-scale genomic analysis and manipulation, in particularly in mice (Lein et al. 2007) and rats.

Finally, as we have argued here, it is imperative to complement experimental studies with the development of full-blown theories that try to capture the essential properties of consciousness; suggest ways to measure them; can be validated through counterintuitive experimental tests; permit the extrapolation to pathological conditions, animals, and even artifacts; and begin to offer a framework to understand how and why the different modalities of consciousness map onto different brain architectures and processes. Only the combination of fine-grained neuronal analysis in mice, rats, and monkeys; ever more sensitive psychophysical and brain imaging techniques in patients and healthy individuals; and the development of a robust theoretical framework can lend hope to the belief that human ingenuity can, ultimately, understand in a rational manner one of the central mysteries of life.

\section{Conflict of Interest}

The authors declare no conflicts of interest.

\section{References}

Abeles, M. (1991). Corticonics Neural circuits of the cerebral cortex. Cambridge, UK, New York: Cambridge University Press.

Alkire, M. T., \& Miller, J. (2005). General anesthesia and the neural correlates of consciousness. Prog. Brain Res., 150, 229-244.
Alkire, M. T., Haier, R. J., \& Fallon, J. H. (2000). Toward a unified theory of narcosis: Brain imaging evidence for a thalamocortical switch as the neurophysiologic basis of anestheticinduced unconsciousness. Conscious Cogn., 9, 370-386.

Alkire, M. T., McReynolds, J. R., Hahn, E. L., \& Trivedi, A. N. (2007). Thalamic microinjection of nicotine reverses sevoflurane-induced loss of righting reflex in the rat. Anesthesiology, 107, 264-272.

Amedi, A., Malach, R., \& Pascual-Leone, A. (2005). Negative BOLD differentiates visual imagery and perception. Neuron, 48, 859-872.

Aravanis, A. M., Wang, L. P., Zhang, F., Meltzer, L. A., Mogri, M. Z., et al. (2007). An optical neural interface: In vivo control of rodent motor cortex with integrated fiberoptic and optogenetic technology. F. Neural Eng., 4, S143-156.

Ascoli, G. A. (1999). Progress and perspectives in computational neuroanatomy. Anat. Rec., 257, 195-207.

Baars, B. J. (1988). A cognitive theory of consciousness. New York: Cambridge University Press.

Baars, B. J. (2005). Global workspace theory of consciousness: Toward a cognitive neuroscience of human experience. Prog. Brain Res., 150, 45-53.

Bachmann, T. (2000). Microgenetic approach to the conscious mind. Amsterdam and Philadelphia: John Benjamins Pub. Co.

Bacon-Mace, N., Mace, M. J., Fabre-Thorpe, M., \& Thorpe, S. J. (2005). The time course of visual processing: Backward masking and natural scene categorisation. Vision Res., 45, 1459-1469.

Bahrami, B., Lavie, N., \& Rees, G. (2007). Attentional load modulates responses of human primary visual cortex to invisible stimuli. Curr. Biol., 17, 509-513.

Barcelo, F., Suwazono, S., \& Knight, R. T. (2000). Prefrontal modulation of visual processing in humans. Nat. Neurosci., 3, 399-403.

Bauby, J. -D. (1997). The diving-bell and the butterfly: A memoir of life in death. New York: Alfred A. Knopf.

Blake, R., \& Logothetis, N. K. (2002). Visual competition. Nat. Rev. Neurosci., 3, 13-21.

Block, N. (2005). Two neural correlates of consciousness. Trends Cogn. Sci., 9, 46-52.

Blumenfeld, H. (2005). Consciousness and epilepsy: Why are patients with absence seizures absent? Prog. Brain Res., 150, 271-286.

Blumenfeld, H., \& Taylor, J. (2003). Why do seizures cause loss of consciousness? Neuroscientist, 9, 301-310.

Blumenfeld, H., McNally, K. A., Vanderhill, S. D., Paige, A. L., Chung, R., et al. (2004). Positive and negative network correlations in temporal lobe epilepsy. Cereb. Cortex, 14, 892-902.

Blumenfeld, H., Westerveld, M., Ostroff, R. B., Vanderhill, S. D., Freeman, J., et al. (2003). Selective frontal, parietal, and temporal networks in generalized seizures. Neuroimage, 19, 1556-1566.

Bower, J. M. (2002). The organization of cerebellar cortical circuitry revisited: implications for function. Ann. N. Y. Acad. Sci., 978, 135-155.

Boyer, J. L., Harrison, S., \& Ro, T. (2005). Unconscious processing of orientation and color without primary visual cortex. Proc. Natl. Acad. Sci. U.S.A., 102, 16875-16879.

Braun, J., \& Sagi, D. (1990). Vision outside the focus of attention. Percept. Psychophys., 48, 45-58. 
Braun, J., \& Julesz, B. (1998). Withdrawing attention at little or no cost: Detection and discrimination tasks. Percept. Psychophys., 60, 1-23.

Braun, J., Koch, C., \& Davis, J. (Eds.). (2001). Visual attention and cortical circuits. Cambridge, MA: MIT Press.

Brecht, M., Singer, W., \& Engel, A. K. (1999). Patterns of synchronization in the superior colliculus of anesthetized cats. 7. Neurosci., 19, 3567-3579.

Buchel, C., Price, C., Frackowiak, R. S., \& Friston, K. (1998). Different activation patterns in the visual cortex of late and congenitally blind subjects. Brain, 121(Pt 3), 409419.

Campagna, J. A., Miller, K. W., \& Forman, S. A. (2003). Mechanisms of actions of inhaled anesthetics. N. Engl. F. Med., $348,2110-2124$.

Chalmers, D. (2000). What is a neural correlate of consciousness? In T. Metzinger (Ed.), Neural correlates of consciousness: Empirical and conceptual questions (pp. 17-40). Cambridge, MA: MIT Press.

Chun, M., \& Wolfe, J. (2000). Visual attention. In E. B. Goldstein (Ed.), Blackwell's handbook of perception (pp. 272-310). Oxford, UK: Blackwell.

Clark, R. E., \& Squire, L. R. (1998). Classical conditioning and brain systems: the role of awareness. Science, 280, 7781.

Cohen, D., \& Yarom, Y. (1998). Patches of synchronized activity in the cerebellar cortex evoked by mossy-fiber stimulation: Questioning the role of parallel fibers. Proc. Natl. Acad. Sci. U.S.A., 95, 15032-15036.

Crick, F., \& Koch, C. (1990). Some reflections on visual awareness. Cold Spring Harbor Symposia on Quantitative Biology, 55, 953-962.

Crick, F., \& Koch, C. (1995). Are we aware of neural activity in primary visual cortex? Nature, 375, 121-123.

Crick, F., \& Koch, C. (1998). Consciousness and neuroscience. Cereb. Cortex, 8, 97-107.

Crick, F., \& Koch, C. (2003). A framework for consciousness. Nat. Neurosci., 6, 119-126.

Damasio, A. R. (1999). The feeling of what happens: Body and emotion in the making of consciousness (1st ed.). New York: Harcourt Brace.

Dehaene, S., Sergent, C., \& Changeux, J. P. (2003). A neuronal network model linking subjective reports and objective physiological data during conscious perception. Proc. Natl. Acad. Sci. U.S.A., 100, 8520-8525.

Dehaene, S., Changeux, J. P., Naccache, L., Sackur, J., \& Sergent, C. (2006). Conscious, preconscious, and subliminal processing: A testable taxonomy. Trends Cogn. Sci., 10, 204 211.

Desimone, R., \& Duncan, J. (1995). Neural mechanisms of selective visual attention. Annual Review of Neuroscience, 18, 193-222.

Destexhe, A., Hughes, S. W., Rudolph, M., \& Crunelli, V. (2007). Are corticothalamic "up" states fragments of wakefulness? Trends Neurosci., 30, 334-342.

Edelman, G. M. (1989). The remembered present: A biological theory of consciousness. New York: BasicBooks.

Edelman, G. M., \& Mountcastle, V. B. (1978). The mindful brain: Cortical organization and the group-selective theory of higher brain function. Cambridge, MA: MIT Press.
Engel, A. K., Fries, P., \& Singer, W. (2001). Dynamic predictions: oscillations and synchrony in top-down processing. Nat. Rev. Neurosci., 2, 704-716.

Felleman, D. J., \& Van Essen, D. C. (1991). Distributed hierarchical processing in the primate cerebral cortex. Cereb. Cortex, $1,1-47$.

Franks, N. P. (2006). Molecular targets underlying general anaesthesia. Br. F. Pharmacol., 147(Suppl 1), S72-81.

Fries, P., Roelfsema, P. R., Engel, A. K., Konig, P., \& Singer, W. (1997). Synchronization of oscillatory responses in visual cortex correlates with perception in interocular rivalry. Proc. Natl. Acad. Sci. U.S.A., 94, 12699-12704.

Fries, P., Neuenschwander, S., Engel, A. K., Goebel, R., \& Singer, W. (2001). Rapid feature selective neuronal synchronization through correlated latency shifting. Nat. Neurosci, 4, 194-200.

Gazzaniga, M. S. (1995). Principles of human brain organization derived from split-brain studies. Neuron, 14, 217-228.

Goodale, M., \& Milner, A. (2005). Sight unseen: An exploration of conscious and unconscious vision. Oxford, UK: Oxford University Press.

Grossberg, S. (1999). The link between brain learning, attention, and consciousness. Conscious Cogn., 8, 1-44.

Guilleminault, C. (1976). Cataplexy. In C. Guilleminault, W. Dennet, \& P. Passouant, (Eds.), Narcolepsy (pp. 125-143). New York: Spectrum.

Han, C. J., O'Tuathaigh, C. M., van Trigt, L., Quinn, J. J., Fanselow, M. S., et al. (2003). Trace but not delay fear conditioning requires attention and the anterior cingulate cortex. Proc. Natl. Acad. Sci. U.S.A., 100, $13087-$ 13092.

Han, X., \& Boyden, E. S. (2007). Multiple-color optical activation, silencing, and desynchronization of neural activity, with single-spike temporal resolution. PLoS ONE, 2, e299.

Hardcastle, V. (1997). Attention versus consciousness: A distinction with a difference. Studies: Bulletin of the Japanese Cognitive Science Society, 4, 56-66.

Hasson, U., Nir, Y., Levy, I., Fuhrmann, G., \& Malach, R. (2004). Intersubject synchronization of cortical activity during natural vision. Science, 303, 1634-1640.

Haynes, J. D., Driver, J., \& Rees, G. (2005). Visibility reflects dynamic changes of effective connectivity between $\mathrm{V} 1$ and fusiform cortex. Neuron, 46, 811-821.

He, S., Cavanagh, P., \& Intriligator, J. (1996). Attentional resolution and the locus of visual awareness. Nature, 383, 334-337.

Hebb, D. O. (1949). The organization of behavior: A neuropsychological theory. New York: Wiley.

Herculano-Houzel, S., Munk, M. H., Neuenschwander, S., \& Singer, W. (1999). Precisely synchronized oscillatory firing patterns require electroencephalographic activation. 7. Neurosci., 19, 3992-4010.

Hobson, J. A., \& Pace-Schott, E. F. (2002). The cognitive neuroscience of sleep: Neuronal systems, consciousness and learning. Nat. Rev. Neurosci., 3, 679-693.

Hobson, J. A., Pace-Schott, E. F., \& Stickgold, R. (2000). Dreaming and the brain: toward a cognitive neuroscience of conscious states. Behav. Brain Sci., 23, 793-842; discussion 904-1121. 
Hollins, M. (1985). Styles of mental imagery in blind adults. Neuropsychologia, 23, 561-566.

Huk, A. C., Ress, D., \& Heeger, D. J. (2001). Neuronal basis of the motion aftereffect reconsidered. Neuron, 32, 161-172.

Hung, C. P., Kreiman, G., Poggio, T., \& DiCarlo, J. J. (2005). Fast readout of object identity from macaque inferior temporal cortex. Science, 310, 863-866.

Imas, O. A., Ropella, K. M., Ward, B. D., Wood, J. D., \& Hudetz, A. G. (2005a). Volatile anesthetics enhance flash-induced gamma oscillations in rat visual cortex. Anesthesiology, 102, 937-947.

Imas, O. A., Ropella, K. M., Ward, B. D., Wood, J. D., \& Hudetz, A. G. (2005b). Volatile anesthetics disrupt frontal-posterior recurrent information transfer at gamma frequencies in rat. Neurosci. Lett., 387, 145-150.

Iwasaki, S. (1993). Spatial attention and two modes of visual consciousness. Cognition, 49, 211-233.

Jiang, Y., Costello, P., Fang, F., Huang, M., \& He, S. (2006). A gender- and sexual orientation-dependent spatial attentional effect of invisible images. Proc. Natl. Acad. Sci. U.S.A., 103, 17048-17052.

Joliot, M., Ribary, U., \& Llinas, R. (1994). Human oscillatory brain activity near $40 \mathrm{~Hz}$ coexists with cognitive temporal binding. Proc. Natl. Acad. Sci. U.S.A., 91, 11748-11751.

Jones, E. G. (1998). A new view of specific and nonspecific thalamocortical connections. Adv. Neurol., 77, 49-71; discussion 72-43.

Kim, C. Y., \& Blake, R. (2005). Psychophysical magic: rendering the visible "invisible." Trends Cogn. Sci., 9, 381-388.

Kirchner, H., \& Thorpe, S. J. (2006). Ultra-rapid object detection with saccadic eye movements: visual processing speed revisited. Vision Res., 46, 1762-1776.

Koch, C., \& Tsuchiya, N. (2007). Attention and consciousness: Two distinct brain processes. Trends Cog. Sci. 11, 16-22.

Kosslyn, S. M., Ganis, G., \& Thompson, W. L. (2001). Neural foundations of imagery. Nat. Rev. Neurosci., 2, 635-642.

Kreiman, G., Fried, I., \& Koch, G. (2002). Single-neuron correlates of subjective vision in the human medial temporal lobe. Proc. Natl. Acad. Sci. U.S.A., 99, 8378-8383.

Lamme, V. A. (2003). Why visual attention and awareness are different. Trends Cogn. Sci., 7, 12-18.

Lamme, V. A. (2006). Towards a true neural stance on consciousness. Trends Cogn. Sci., 10, 494-501.

Lamme, V. A., \& Roelfsema, P. R. (2000). The distinct modes of vision offered by feedforward and recurrent processing. Trends Neurosci., 23, 571-579.

Langston, J., \& Palfreman, J. (1995). The case of the frozen addicts. New York: Vintage Books.

Laureys, S., Owen, A. M., \& Schiff, N. D. (2004). Brain function in coma, vegetative state, and related disorders. Lancet Neurol., 3, 537-546.

Laureys, S., Faymonville, M. E., Luxen, A., Lamy, M., Franck, G., et al. (2000a). Restoration of thalamocortical connectivity after recovery from persistent vegetative state. Lancet, 355, 1790-1791.

Laureys, S., Faymonville, M. E., Degueldre, C., Fiore, G. D., Damas, P., et al. (2000b). Auditory processing in the vegetative state. Brain, 123(Pt 8), 1589-1601.

Laureys, S., Faymonville, M. E., Peigneux, P., Damas, P., Lambermont, B., et al. (2002). Cortical processing of noxious somatosensory stimuli in the persistent vegetative state. Neuroimage, 17, 732-741.

Laureys, S., Pellas, F., Van Eeckhout, P., Ghorbel, S., Schnakers, C., et al. (2005). The locked-in syndrome: What is it like to be conscious but paralyzed and voiceless? Prog. Brain Res., 150, 495-511.

Lee, S. H., Blake, R., \& Heeger, D. J. (2005). Traveling waves of activity in primary visual cortex during binocular rivalry. Nat. Neurosci, 8, 22-23.

Lee, S. H., Blake, R., \& Heeger, D. J. (2007). Hierarchy of cortical responses underlying binocular rivalry. Nat. Neurosci, 10, 1048-1054.

Lein, E. S., et al. (2007). Genome-wide atlas of gene expression in the adult mouse brain. Nature, 445, 168-176.

Leopold, D. A., \& Logothetis, N. K. (1996). Activity changes in early visual cortex reflect monkeys' percepts during binocular rivalry. Nature, 379, 549-553.

Li, F. F., VanRullen, R., Koch, C., \& Perona, P. (2002). Rapid natural scene categorization in the near absence of attention. Proc. Natl. Acad. Sci. U.S.A., 99, 9596-9601.

Logothetis, N. K. (1998). Single units and conscious vision. Philos. Trans. R. Soc. Lond. B Biol. Sci., 353, 1801-1818.

Lorente de Nó, R. (1938). The cerebral cortex: Architecture, intracortical connections and motor projections. In J. Fulton (Ed.), Physiology of the nervous system (pp. 291-339). London: Oxford University Press.

Lumer, E. D. (1998). A neural model of binocular integration and rivalry based on the coordination of action-potential timing in primary visual cortex. Cereb. Cortex, 8, 553561.

Lumer, E. D., Edelman, G. M., \& Tononi, G. (1997a). Neural dynamics in a model of the thalamocortical system . 2. The role of neural synchrony tested through perturbations of spike timing. Cerebral Cortex, 7, 228-236.

Lumer, E. D., Edelman, G. M., \& Tononi, G. (1997b). Neural dynamics in a model of the thalamocortical system. 1. Layers, loops and the emergence of fast synchronous rhythms. Cerebral Cortex, 7, 207-227.

Mack, A., \& Rock, I. (1998). Inattentional blindness. Cambridge, MA: MIT Press.

Macknik, S. L. (2006). Visual masking approaches to visual awareness. Prog. Brain Res., 155, 177-215.

Maquet, P., Péters, J., Aerts, J., Delfiore, G., Degueldre, C., et al. (1996). Functional neuroanatomy of human rapideye-movement sleep and dreaming. Nature, 383, 163-166.

Markowitsch, H. J., \& Kessler, J. (2000). Massive impairment in executive functions with partial preservation of other cognitive functions: The case of a young patient with severe degeneration of the prefrontal cortex. Exp. Brain Res., 133, 94-102.

Markram, H. (2006). The blue brain project. Nat. Rev. Neurosci., 7, 153-160.

Martinez-Conde, S., Macknik, S. L., \& Hubel, D. H. (2002). The function of bursts of spikes during visual fixation in the awake primate lateral geniculate nucleus and primary visual cortex. Proc. Natl. Acad. Sci. U.S.A., 99, 13920 13925.

Martinez-Conde, S., Macknik, S. L., \& Hubel, D. H. (2004). The role of fixational eye movements in visual perception. Nat. Rev. Neurosci., 5, 229-240. 
Martinez-Conde, S., Macknik, S. L., Troncoso, X. G., \& Dyar, T. A. (2006). Microsaccades counteract visual fading during fixation. Neuron, 49, 297-305.

Martinez-Conde, S., Cudeiro, J., Grieve, K. L., Rodriguez, R., Rivadulla, C., et al. (1999). Effects of feedback projections from area 18 layers $2 / 3$ to area 17 layers $2 / 3$ in the cat visual cortex. F. Neurophysiol., 82, 2667-2675.

Mason, M. F., Norton, M. I., Van Horn, J. D., Wegner, D. M., Grafton, S. T., et al. (2007). Wandering minds: The default network and stimulus-independent thought. Science, 315, 393-395.

Massimini, M., Huber, R., Ferrarelli, F., Hill, S., et al. (2004). The sleep slow oscillation as a traveling wave. F. Neurosici, $24,6862-6870$.

Massimini, M., Ferrarelli, F., Huber, R., Esser, S. K., Singh, H., et al. (2005). Breakdown of cortical effective connectivity during sleep. Science, 309, 2228-2232.

Massimini, M., Ferrarelli, F., Esser, S. K., Riedner, B. A., Huber, R., et al. (2007). Triggering sleep slow waves by transcranial magnetic stimulation. Proc. Natl. Acad. Sci. U.S.A., 104, 8496-8501.

Mataro, M., Jurado, M. A., Garcia-Sanchez, C., Barraquer, L., Costa-Jussa, F. R., et al. (2001). Long-term effects of bilateral frontal brain lesion: 60 years after injury with an iron bar. Arch. Neurol., 58, 1139-1142.

Melloni, L., Molina, C., Pena, M., Torres, D., Singer, W., et al. (2007). Synchronization of neural activity across cortical areas correlates with conscious perception. F. Neurosci., 27, 2858-2865.

Merikle, P. M., \& Joordens, S. (1997). Parallels between perception without attention and perception without awareness. Conscious Cogn., 6, 219-236.

Miller, J. W., \& Ferrendelli, J. A. (1990). Characterization of GABAergic seizure regulation in the midline thalamus. Neuropharmacology, 29, 649-655.

Miller, J. W., Hall, C. M., Holland, K. D., \& Ferrendelli, J. A. (1989). Identification of a median thalamic system regulating seizures and arousal. Epilepsia, 30, 493-500.

Montaser-Kouhsari, L., \& Rajimehr, R. (2004). Attentional modulation of adaptation to illusory lines. F. Vis., 4, 434-444.

Munk, M. H., Roelfsema, P. R., Konig, P., Engel, A. K., \& Singer, W. (1996). Role of reticular activation in the modulation of intracortical synchronization. Science, 272, $271-$ 274.

Naccache, L., Blandin, E., \& Dehaene, S. (2002). Unconscious masked priming depends on temporal attention. Psychol. Sci., 13, 416-424.

Nakakimura, K., Sakabe, T., Funatsu, N., Maekawa, T., \& Takeshita, H. (1988). Metabolic activation of intercortical and corticothalamic pathways during enflurane anesthesia in rats. Anesthesiology, 68, 777-782.

O'Regan, J. K., \& Noe, A. (2001). A sensorimotor account of vision and visual consciousness. Behav. Brain Sci., 24, 939 973; discussion 973-1031.

Ori, G., Dam, M., Pizzolato, G., Battistin, L., \& Giron, G. (1986). Effects of isoflurane anesthesia on local cerebral glucose utilization in the rat. Anesthesiology, 65, 152-156.

Owen, A. M., Coleman, M. R., Boly, M., Davis, M. H., Laureys, S., et al. (2006). Detecting awareness in the vegetative state. Science, 313, 1402.
Pascual-Leone, A., \& Walsh, V. (2001). Fast backprojections from the motion to the primary visual area necessary for visual awareness. Science, 292, 510-512.

Pashler, H. (1998). The psychology of attention. Cambridge, MA: MIT Press.

Poppel, E., \& Artin, T. (1988). Mindworks: Time and conscious experience. Boston: Harcourt Brace Jovanovich.

Posner, M. I. (1994). Attention: The mechanisms of consciousness. Proc. Nat. Acad. Sci. U.S.A., 91, 7398-7403.

Raymond, J. E., Shapiro, K. L., \& Arnell, K. M. (1992). Temporary suppression of visual processing in an RSVP task: An attentional blink? 7. Exp. Psychol. Hum. Percept. Perform., 18, 849-860.

Reddy, L., \& Koch, C. (2006). Face identification in the nearabsence of focal attention. Vision Res., 46, 2336-2343.

Reddy, L., Wilken, P., \& Koch, C. (2004). Face-gender discrimination is possible in the near-absence of attention. F. Vis., 4, 106-117.

Rees, G., \& Frith, C. (2007). Methodologies for identifying the neural correlates of consciousness. In M. Velmans \& S. Schneider (Eds.), The Blackwell companion to consciousness (pp. 553-566). Oxford, UK: Blackwell.

Ro, T., Breitmeyer, B., Burton, P., Singhal, N. S., \& Lane, D. (2003). Feedback contributions to visual awareness in human occipital cortex. Curr. Biol., 13, 10381041.

Roelfsema, P. R., Engel, A. K., Konig, P., \& Singer, W. (1997). Visuomotor integration is associated with zero time-lag synchronization among cortical areas. Nature, 385, 157161.

Rolls, E. T., Tovee, M. J., \& Panzeri, S. (1999). The neurophysiology of backward visual masking: information analysis. 7. Cogn. Neurosci., 11, 300-311.

Rossi, A. F., Desimone, R., \& Ungerleider, L. G. (2001). Contextual modulation in primary visual cortex of macaques. $\mathcal{F}$. Neurosci., 21, 1698-1709.

Schiff, N. D. (2006a). Multimodal neuroimaging approaches to disorders of consciousness. F. Head Trauma Rehabil., 21, 388-397.

Schiff, N. D. (2006b). Measurements and models of cerebral function in the severely injured brain. F. Neurotrauma, 23, 1436-1449.

Schiff, N. D., Giacino, J. T., Kalmar, K., Victor, J. D., Baker, K., et al. (2007). Behavioural improvements with thalamic stimulation after severe traumatic brain injury. Nature, 448, 600-603.

Schoffelen, J. M., Oostenveld, R., \& Fries, P. (2005). Neuronal coherence as a mechanism of effective corticospinal interaction. Science, 308, 111-113.

Scholte, H. S., Witteveen, S. C., Spekreijse, H., \& Lamme, V. A. (2006). The influence of inattention on the neural correlates of scene segmentation. Brain Res., 1076, 106 115 .

Sergent, C., Baillet, S., \& Dehaene, S. (2005). Timing of the brain events underlying access to consciousness during the attentional blink. Nat. Neurosci., 8, 1391-1400.

Sheinberg, D. L., \& Logothetis, N. K. (1997). The role of temporal cortical areas in perceptual organization. Proc. Natl. Acad. Sci. U.S.A., 94, 3408-3413.

Siegel, J. (2000). Narcolepsy. Sci. Am., 282, 76-81. 
Silvanto, J., Cowey, A., Lavie, N., \& Walsh, V. (2005). Striate cortex (V1) activity gates awareness of motion. Nat. Neurosci., 8, 143-144.

Singer, W. (1999). Neuronal synchrony: A versatile code for the definition of relations? Neuron, 24, 49-65, 111125.

Singer, W., \& Gray, C. M. (1995). Visual feature integration and the temporal correlation hypothesis. Ann. Rev. Neurosci., 18, 555-586.

Sperling, G., \& Dosher, B. (1986). Strategy and optimization in human information processing. In K. Boff, L. Kaufman, \& J. Thomas (Eds.), Handbook of perception and human performance (pp. 1-65). New York: Wiley.

Steriade, M. (2003). The corticothalamic system in sleep. Front. Biosci., 8, D878-899.

Steriade, M., Timofeev, I., \& Grenier, F. (2001). Natural waking and sleep states: a view from inside neocortical neurons. 7. Neurophysiol., 85, 1969-1985.

Super, H., Spekreijse, H., \& Lamme, V. A. (2001). Two distinct modes of sensory processing observed in monkey primary visual cortex (V1). Natl. Neurosci., 4, 304 310.

Thorpe, S., Fize, D., \& Marlot, C. (1996). Speed of processing in the human visual system. Nature, 381, 520522.

Tong, F. (2003). Primary visual cortex and visual awareness. $\mathcal{N}$ at. Rev. Neurosci., 4, 219-229.

Tong, F., Nakayama, K., Vaughan, J. T., \& Kanwisher, N. (1998). Binocular rivalry and visual awareness in human extrastriate cortex. Neuron, 21, 753-759.

Tononi, G. (2004a). Consciousness and the brain: Theoretical aspects. In G. Adelman \& B. Smith (Eds.), Encyclopedia of neuroscience (3rd ed.). Elsevier.

Tononi, G. (2004b). An information integration theory of consciousness. BMC Neurosci., 5, 42.

Tononi, G. (2005). Consciousness, information integration, and the brain. Prog. Brain Res., 150, 109-126.

Tononi, G., \& Edelman, G. M. (1998). Consciousness and complexity. Science, 282, 1846-1851.

Tononi, G., Sporns, O., \& Edelman, G. M. (1992). Reentry and the problem of integrating multiple cortical areas: Simu- lation of dynamic integration in the visual system. Cereb. Cortex, 2, 310-335.

Tononi, G., Sporns, O., \& Edelman, G. M. (1996). A complexity measure for selective matching of signals by the brain. Proc. Natl. Acad. Sci. U.S.A., 93, 3422-3427.

Tse, P. U., Martinez-Conde, S., Schlegel, A. A., \& Macknik, S. L. (2005). Visibility, visual awareness, and visual masking of simple unattended targets are confined to areas in the occipital cortex beyond human V1/V2. Proc. Natl. Acad. Sci. U.S.A., 102, 17178-17183.

Tsuchiya, N., \& Koch, C. (2005). Continuous flash suppression reduces negative afterimages. Nat. Neurosci., 8, 1096-1101.

Vahle-Hinz, C., Detsch, O., Siemers, M., \& Kochs, E. (2007). Contributions of GABAergic and glutamatergic mechanisms to isoflurane-induced suppression of thalamic somatosensory information transfer. Exp. Brain Res., 176, 159-172.

VanRullen, R., \& Koch, C. (2003). Visual selective behavior can be triggered by a feed-forward process. f. Cogn. Neurosci., 15, 209-217.

Velly, L. J., Rey, M. F., Bruder, N. J., Gouvitsos, F. A., Witjas, T., et al. (2007). Differential dynamic of action on cortical and subcortical structures of anesthetic agents during induction of anesthesia. Anesthesiology, 107, 202-212.

Vogel, E. K., Luck, S. J., \& Shapiro, K. L. (1998). Electrophysiological evidence for a postperceptual locus of suppression during the attentional blink. F. Exp. Psychol. Hum. Percept. Perform., 24, 1656-1674.

Voss, H. U., Uluc, A. M., Dyke, J. P., Watts, R., Kobylarz, E. J., et al. (2006). Possible axonal regrowth in late recovery from the minimally conscious state. F. Clin. Invest., 116, 2005-2011.

Wolfe, J. M. (1984). Reversing ocular dominance and suppression in a single flash. Vision Res., 24, 471-478.

Womelsdorf, T., Schoffelen, J. M., Oostenveld, R., Singer, W., Desimone, R., et al. (2007). Modulation of neuronal interactions through neuronal synchronization. Science, 316 , 1609-1612.

Zhang, F., Wang, L. P., Brauner, M., Liewald, J. F., Kay, K., et al. (2007). Multimodal fast optical interrogation of neural circuitry. Nature, 446, 633-639. 